\title{
A new CFA interpolation framework
}

\author{
Rastislav Lukac*, Konstantinos N. Plataniotis, Dimitrios Hatzinakos, \\ Marko Aleksic
}

The Edward S. Rogers Sr. Department of ECE, University of Toronto, 10 King's College Road, Toronto, Ont., Canada M5S $3 G 4$

Received 15 September 2003; received in revised form 30 August 2004

Available online 5 October 2005

\begin{abstract}
The paper introduces a new color filter array (CFA) interpolation method for digital still cameras. The proposed interpolation scheme is able to (i) overcome the hardware limitations of existing CFA based image acquisition solutions, and (ii) restore color images with excellent visual quality. The scheme employs an adaptive edge-sensing mechanism which operates along the vertical, horizontal and diagonal directions to correctly interpolate unavailable color components. Building on the computed edge-sensing map and a refined color-difference model, a new correlation-correction algorithm is introduced. In addition to the basic model, adaptively determined correction operations are also discussed and analyzed. The solutions proposed here, described in a novel vector notation, constitute a unique CFA interpolation framework, which readily unifies previous, seemingly unrelated, results. Simulation studies indicate that the proposed method is computationally efficient and yields excellent performance, in terms of subjective and objective image quality measures, while outperforming state-of-the-art CFA interpolation methods.
\end{abstract}

(C) 2005 Elsevier B.V. All rights reserved.

Keywords: Digital camera; Color filter array; Bayer pattern; Demosaicing; Image restoration; Color interpolation

\section{Introduction}

Color image processing [1-3] has aroused much interest and acclaim over the past few years. The advances in hardware and software platforms have allowed capturing and reproducing of real scenes in color as never before. Digital cameras for still images are among the most popular acquisition devices, whose commercial proliferation has a significant impact on the research in this area.

\footnotetext{
*Corresponding author. Tel.: + 14169786845 ; fax: +14169784425 .

E-mail address: lukacr@dsp.utoronto.ca (R. Lukac).

$U R L$ : http://www.dsp.utoronto.ca/ lukacr.
}

Digital color cameras acquire color information by transmitting the image through Red (R), Green $(\mathrm{G})$ and Blue (B) color filters having different spectral transmittances and then sampling the resulted images using three electronic sensors (Fig. 1a) [3], usually charge-coupled devices (CCD) and complementary metal oxide semiconductor (CMOS) sensors. To reduce cost and complexity, digital camera manufacturers use a single CCD/CMOS sensor (Fig. 1b) with a color filter array (CFA) to capture all the three primary colors $(\mathrm{R}, \mathrm{G}, \mathrm{B})$ at the same time [4-6]. The Bayer pattern (Fig. 2) [7], a widely used CFA, provides the array or mosaic of the RGB colors so that only one color element is available in each pixel, whereas two missing colors must be estimated from the adjacent 
(a)

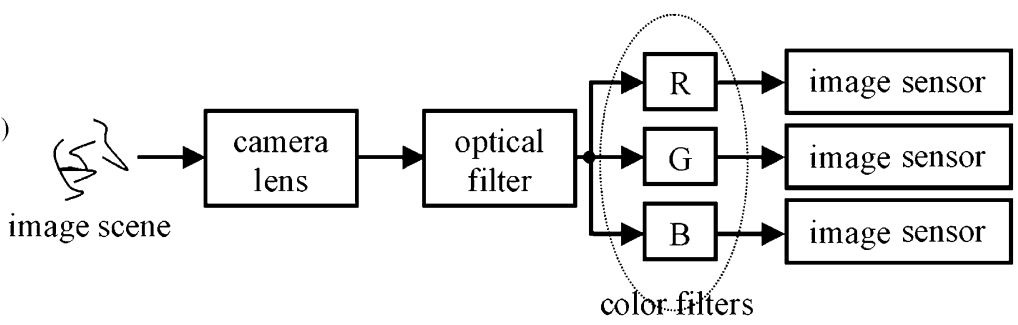

(b)

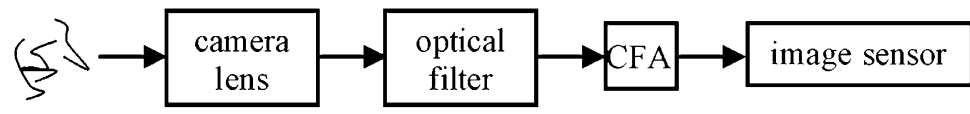

image scene

Fig. 1. Image acquisition process using: (a) a three-sensor device, (b) a single-sensor device.

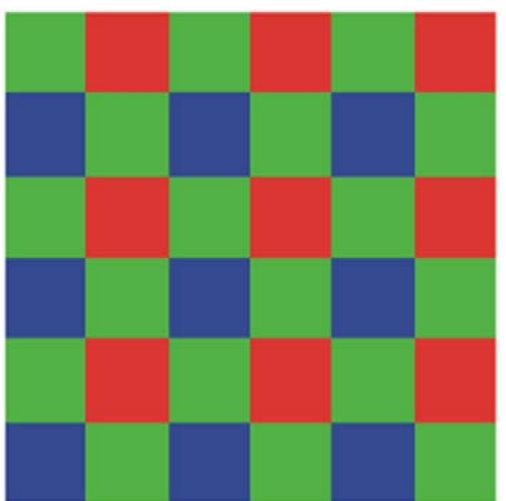

Fig. 2. Bayer CFA pattern.

pixels. This process is called CFA interpolation, or demosaicing.

CFA interpolation algorithms are usually classified into:

- Non-adaptive interpolation techniques [8-10], which attempt image restoration regardless of the structural contents in the images.

- Edge-adaptive interpolation techniques [11-13], which incorporate some kind of structural information into the image restoration process.

Although non-adaptive interpolation techniques are computationally efficient, their performance is inefficient as the omission of edge information during the restoration process often results in image blurring. Although some non-adaptive techniques, e.g. $[9,10]$ are engineered to achieve excellent results in terms of objective evaluation of image quality, they often impair visual image quality near fine edges and structural details.
Edge-adaptive interpolation methods have been considered in order to improve the signal-detail preserving capability of the restoration process. The inclusion of an edge detection step provides high accuracy in estimating and restoring edges and high frequency components in the image. There are at least three possible ways to utilize edge information in the interpolation process. The first approach employs edge detection to guide the interpolation process [14,15], which makes the method computationally expensive. Another approach takes advantage of an implicit edge detection, step which is performed based on user-defined parameters [16]. This parameter setting often decreases the robustness of the methods. Finally, a fully adaptive edge detection scheme can be incorporated into the interpolation process $[11,13,17]$. It should be noted at this point that the improvement in terms of performance comes at the expense of the computational simplicity of the algorithm. It is evident that the edge detection step slows down the restoration process increasing the computational complexity of the solution.

The proposed method here is a fully adaptive, implicit edge-detection based interpolation scheme. To improve the color appearance and enhance perception of the structural contents, the proposed interpolation scheme employs a correction step. The introduced correction mechanism operates on the so-called color-difference model [18] by taking advantage of the correlation between the RGB channels of natural images.

It is evident that the employed correction process is useful in highly correlated images or image areas with a number of high-frequency transitions. However, in images with a globally week correlation 
and/or high local variations in correlation amongst the RGB channels, the correlation-correction step is simply unnecessary or counter productive. Therefore, two extensions of our basic idea [4] are introduced. Both solutions constrain the correction process depending on the estimated degree of correlation between the color channels under consideration. In this paper, we will make use of a global correlation approach, which bypasses/engages the correction process based on information gathered from the image as a whole. A second variation of the proposed method utilizes a correlation map or local image statistics to accommodate the correction process.

The proposed basic concept along with the abovementioned modifications constitute a unique CFA interpolation framework which avoids edge blurring while improving on the color appearance obtained from previous interpolation techniques. This edge-sensing correlation-correction (ESCC) framework interpolates the missing RGB components along edge directions and significantly reduces the level of aliasing artifacts present in the restored output compared to the prior art. The incorporated correction process is capable of improving the color appearance in most natural images, which results in restored images with excellent fidelity.

Lastly, it should be noted at this point that a novel vector notation is used to describe the CFA interpolation process. The vector notation, commonly used in color image processing tasks $[1,2]$ is tapped here to assist with the development of a compact CFA framework.

The rest of the paper is organized as follows. In Section 2, the formulation of the problem is introduced and main features of the state of the art in CFA interpolation are presented. The proposed CFA framework is introduced in Section 3. Motivation and design characteristics are discussed in detail and variations of the proposed methods are provided and analyzed with respect to their properties and parameters used. In Section 4, the proposed framework is tested using a variety of test images. Extended simulation studies are included in order to demonstrate the effectiveness of the proposed schemes and comparisons in terms of performance with the state of the art are provided. Performance comparison are given in terms of commonly used image quality measures. Finally, this paper concludes in Section 5.

\section{Problem formulation}

The CFA interpolation relates to the problem of restoration of subsampled color images. Bayer pattern demosaicing is fundamental to the operation of most of current single-sensor digital cameras. Since the notation of CFA interpolation remains relatively unknown, a brief overview to the problem and the state of the art is included for completeness.

All CFA interpolation methods developed in the past few years are based on the notion of a supporting sliding window. These methods utilize a variety of estimation operators [19-21], correlation models $[18,22,23]$ and edge-sensing mechanisms [11-13,17]. Therefore, not surprising that, they have different computational complexity requirement and wide variations in performance [24-28].

Let us consider, a $K_{1} \times K_{2}$ gray-scale image $z(l)$ : $Z^{2} \rightarrow Z$ representing a two-dimensional matrix of integer samples. In the Bayer CFA pattern, half of the pixels $z_{l} \in Z^{2}$, for $l=1,2, \ldots, K_{1} K_{2}$, correspond to the $\mathrm{G}$ channel, whereas other signals such as the R,B channels populate the other half. The $G$ channel contains the most important information for the color image as perceived by humans. Assuming that $p=1,2, \ldots, K_{1}$ and $q=1,2, \ldots, K_{2}$ denote the spatial position of the pixels in vertical (image rows) and horizontal (image columns) directions shown in Fig. 3, gray-scale pixels $z_{l}$, for $l=(p-1) K_{2}+q$, can be transformed into the RGB vectors $\mathbf{x}_{l}=\left(x_{l 1}, x_{l 2}, x_{l 3}\right) \in Z^{2}$ as follows:

$\mathbf{x}_{l}= \begin{cases}\left(z_{l}, 0,0\right) & \text { for } p \text { odd and } q \text { even, } \\ \left(0,0, z_{l}\right) & \text { for } p \text { even and } q \text { odd } \\ \left(0, z_{l}, 0\right) & \text { otherwise }\end{cases}$

This transformation forms a $K_{1} \times K_{2}$ RGB image $\mathbf{x}(l): Z^{2} \rightarrow Z^{3}$ representing the two-dimensional matrix of three-component samples (Fig. 3). Note that the color vectors $\mathbf{x}_{l}$ correspond to one true component $x_{l k}$, as it varies from position to position, whereas the two components of $\mathbf{x}_{l}$ are set to zero.

The objective of the interpolation process is to estimate the missing color components of $\mathbf{x}(l)$ and reconstitute the interpolated $\mathrm{RGB}$ image $\mathbf{y}(l)$ : $Z^{2} \rightarrow Z^{3}$ to be as close as possible to the desired RGB image $\mathbf{o}(l): Z^{2} \rightarrow Z^{3}$. As in most image processing problems, a "loss function," which depends on the unavailable full RGB sample and the interpolation vector, is used to penalize errors 

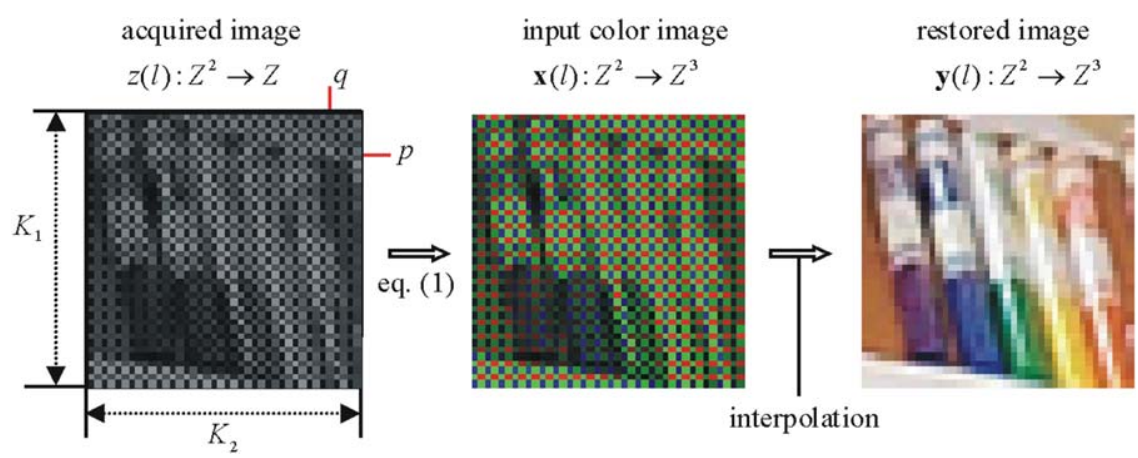

Fig. 3. Color image restoration process.

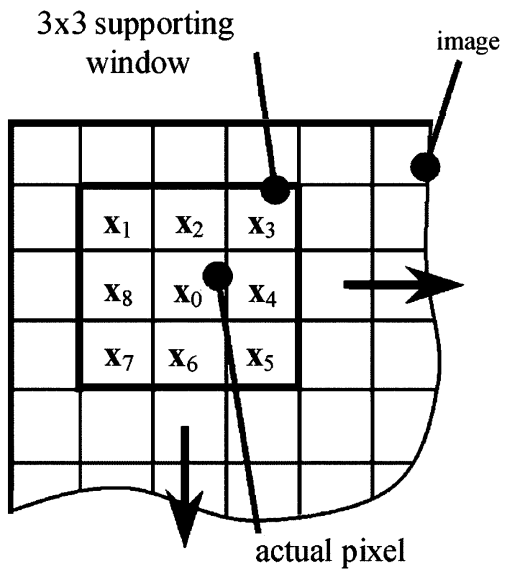

Fig. 4. Indexing of the samples inside a $3 \times 3$ sliding supporting window $W$.

during the procedure. It is natural to assume that if one penalizes interpolation errors through loss function, then the optimal solution is the function of the inputs that minimizes the expected average loss:

minimize $E\left\{\|\mathbf{o}-\mathbf{y}\|^{2}\right\}$,

where $E\{\cdot\}$ indicates statistical expectation guaranteeing the minimum average loss or risk [29].

To estimate the missing color components of $\mathbf{x}(l)$, a supporting window $W=\left\{\mathbf{x}_{i} \in Z^{2} ; i=0,1, \ldots\right.$, $N-1\}$ of finite size $N$ is considered with the sample $\mathbf{x}_{0}$ placed in the center of the window (Fig. 4). This window-operator slides over the image to affect individually all the image pixels and changes the center $\mathbf{x}_{0}$ by some function of the local neighborhood area $\left\{\mathbf{x}_{1}, \mathbf{x}_{2}, \ldots, \mathbf{x}_{N-1}\right\}$ at a time. The rationale of this approach is to minimize the local distortion and ensure the stationarity of the processes generating the image.

\section{Proposed CFA interpolation framework}

Many developed CFA methods do not utilize any edge-sensing support $[9,20,30]$ or operate only in horizontal/vertical directions [11,16,31]. This leads to false colors and perceived blurring, since these methods do not follow complete structural information of the image. Therefore, some recently introduced schemes, e.g. $[12,13,17]$ operate in 8 directions and produce high-quality images. These methods perform repeated interpolations of the $G$ values without improving the accuracy of the $\mathrm{R}, \mathrm{B}$ values. Introducing the edge-sensing correction mechanism into the R,B channels could improve the visual quality of restored images.

\subsection{Edge-detection process}

To follow the edges in all possible directions, i.e. diagonal, horizontal and vertical edges, the proposed method employs eight-direction mechanism of [17]. Calculation of edge information requires a $5 \times 5$ supporting window moving over the image domain $z(l): Z^{2} \rightarrow Z$ (Fig. 5). As a result, a diagonal edge in top-left direction is quantified by the weight

$w_{1}=\frac{1}{1+\left(\left|z_{0}-z_{9}\right|+\left|z_{1}-z_{5}\right|\right) /(2 \sqrt{2})}$,

where $z_{0}$ is the reference pixel related to the same color diagonally positioned in $z_{9}$, whereas $z_{1}$ and $z_{5}$ denote another color pair in the same top-left direction. Others diagonal edges reflect to the quantities analogously expressed as follows:

$w_{3}=\frac{1}{1+\left(\left|z_{0}-z_{13}\right|+\left|z_{3}-z_{7}\right|\right) /(2 \sqrt{2})}$, 


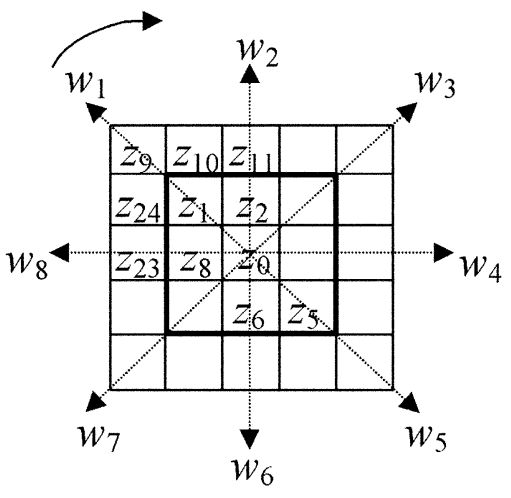

Fig. 5. Association of the edge-sensing weight coefficients.

$$
\begin{aligned}
& w_{5}=\frac{1}{1+\left(\left|z_{0}-z_{17}\right|+\left|z_{5}-z_{1}\right|\right) /(2 \sqrt{2})}, \\
& w_{7}=\frac{1}{1+\left(\left|z_{0}-z_{21}\right|+\left|z_{7}-z_{3}\right|\right) /(2 \sqrt{2})},
\end{aligned}
$$

where $w_{3}, w_{5}$ and $w_{7}$ corresponds to the edges in top-right, bottom-right and bottom-left directions, respectively.

Sensing edge information in horizontal and vertical directions, the corresponding quantities are given by

$$
\begin{aligned}
& w_{2}=\frac{1}{1+\left(\left|z_{0}-z_{11}\right|+\left|z_{2}-z_{6}\right|\right) / 2}, \\
& w_{4}=\frac{1}{1+\left(\left|z_{0}-z_{15}\right|+\left|z_{4}-z_{8}\right|\right) / 2}, \\
& w_{6}=\frac{1}{1+\left(\left|z_{0}-z_{19}\right|+\left|z_{6}-z_{2}\right|\right) / 2}, \\
& w_{8}=\frac{1}{1+\left(\left|z_{0}-z_{23}\right|+\left|z_{8}-z_{4}\right|\right) / 2},
\end{aligned}
$$

where $w_{2}$ and $w_{6}$ correspond to edge information in vertical top and bottom directions, respectively; whereas $w_{4}$ and $w_{8}$ reflect the edges in horizontal right and left directions, respectively. Note that the absolute differences in (3) to (10) are useful in terms of implementation efficiency [17], however, they can be replaced with squared differences.

\subsection{Interpolation process}

In the next step, the weight coefficients $w_{1}, w_{2}, \ldots, w_{N-1}$ are utilized in the interpolation of the $\mathrm{G}$ channel. Note that the interpolation process utilizes a $3 \times 3$ window $(N=9)$ sliding over the color image $\mathbf{x}(l): Z^{2} \rightarrow Z^{3}$. This edge-adaptive estimation of the $\mathrm{G}$ channel is given by

$\bar{y}_{02}= \begin{cases}x_{02} & \text { if } z_{0} \cong x_{02}, \\ \sum_{i=1}^{N-1} w_{i}^{\prime} x_{i 2}^{\prime} & \text { otherwise, }\end{cases}$

where $z_{0}$ is the acquired pixel before the transformation (1) in the same spatial position as the color (RGB) vector $\mathbf{x}_{0}$, operator $\cong$ denotes a one to one relationship and $i$ denotes the compass orientation clockwise (Fig. 5). Normalized weight coefficient $w_{i}^{\prime}=w_{i} / \sum_{j=1}^{N-1} w_{j}$ measures the importance of the predicted green neighbor $x_{i 2}^{\prime}$ based on local edge information. Similarly, as for edge information, this calculation differentiate the samples in diagonal and vertical/horizontal directions [17]. Diagonally positioned samples have predicted $\mathrm{G}$ values obtained as follows:

$x_{(1) 2}^{\prime}=\frac{x_{(8) 2}+x_{(2) 2}+\left(z_{1}-z_{5}\right) /(2 \sqrt{2})+\left(z_{23}-z_{0}+z_{11}-z_{0}\right) / 4}{2}$,

$x_{(3) 2}^{\prime}=\frac{x_{(2) 2}+x_{(4) 2}+\left(z_{3}-z_{7}\right) /(2 \sqrt{2})+\left(z_{11}-z_{0}+z_{15}-z_{0}\right) / 4}{2}$,

$x_{(5) 2}^{\prime}=\frac{x_{(4) 2}+x_{(6) 2}+\left(z_{5}-z_{1}\right) /(2 \sqrt{2})+\left(z_{15}-z_{0}+z_{19}-z_{0}\right) / 4}{2}$,

$x_{(7) 2}^{\prime}=\frac{x_{(6) 2}+x_{(8) 2}+\left(z_{7}-z_{3}\right) /(2 \sqrt{2})+\left(z_{23}-z_{0}+z_{19}-z_{0}\right) / 4}{2}$,

whereas vertically/horizontally positioned samples have the $G$ channel values predicted by

$x_{(2) 2}^{\prime}=x_{(2) 2}+\left(z_{11}-z_{0}+z_{2}-z_{6}\right) / 4$,

$x_{(4) 2}^{\prime}=x_{(4) 2}+\left(z_{15}-z_{0}+z_{4}-z_{8}\right) / 4$,

$x_{(6) 2}^{\prime}=x_{(6) 2}+\left(z_{19}-z_{0}+z_{6}-z_{2}\right) / 4$,

$x_{(8) 2}^{\prime}=x_{(8) 2}+\left(z_{23}-z_{0}+z_{8}-z_{4}\right) / 4$.

Assuming that the R,B channels are highly correlated to the $G$ channel, more accurate $G$ values of (11) can be utilized in the color-difference model [18]. This increases the estimation accuracy of the corresponding R,B values. The procedure (the 3rd and 4th steps in Fig. 6) can be 


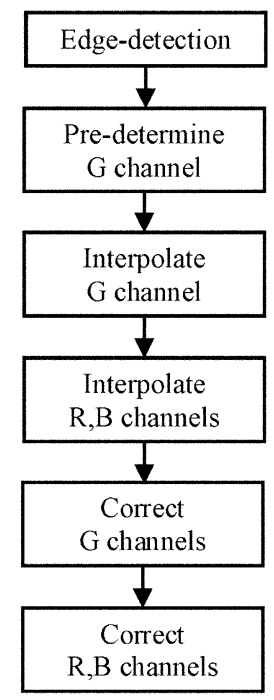

Fig. 6. Body of the proposed CFA interpolation framework.

described as follows:

$$
\bar{y}_{0 k}=\left\{\begin{aligned}
x_{0 k} & \text { if } z_{0} \cong x_{0 k}, \\
\bar{y}_{02}+\sum_{i=1}^{(N-1) / 2} w_{i}^{\prime \prime} & \\
\times\left(x_{(2 i) k}-\bar{y}_{(2 i) 2}\right) & \text { if } z_{0} \cong x_{02}, \\
\bar{y}_{02}+\sum_{i=1}^{(N-1) / 2} w_{i}^{\prime \prime \prime} & \\
\times\left(x_{(2 i-1) k}-\bar{y}_{(2 i-1) 2}\right) & \text { if } z_{0} \cong x_{0(k \pm 2),}
\end{aligned}\right.
$$

where $k=1$ and $k=3$ characterize the $\mathrm{R}$ and $\mathrm{B}$ channels, respectively, and $w_{i}^{\prime \prime}=w_{2 i} / \sum_{j=1}^{(N-1) / 2} w_{2 j}$ are the normalized weights corresponding to edges in north, east, south and west directions, whereas $w_{i}^{\prime \prime \prime}=w_{(2 i-1)} / \sum_{j=1}^{(N-1) / 2} w_{(2 j-1)}$ are the weights corresponding to the diagonally positioned edges.

\subsection{Correction process}

It is known that the human visual system is sensitive to changes in color and edge information, which provides indication of the shape of objects in the image [1,2]. False colored and blurred edges introduced as result of inaccurate interpolation significantly degrade the quality of the perceived image. Introducing a correction mechanism into the interpolation process improves contrast and chrominance properties of the initially interpolated $G$ channel $[13,17]$.
The proposed method utilizes the correlation-correction step based on the correlation amongst the RGB channels and support of initially computed edge information. Since popular saturation models fails in image areas when the $G$ values are too small (dividing by zero or very small numbers shifts the saturation models out of work), we make use of a simple color difference model. Thus, the correction process related to the $\mathrm{G}$ channel is given by

$y_{02}= \begin{cases}\bar{y}_{0 k}+\sum_{i=1}^{(N-1) / 2} w_{i}^{\prime \prime}\left(\bar{y}_{(2 i) 2}-\bar{y}_{(2 i) k}\right) & \text { if } z_{0} \cong x_{0 k}, \\ \bar{y}_{02} & \text { otherwise, }\end{cases}$

where $w_{i}^{\prime \prime}$ are the same weights as in (20).

Considering the corrected $G$ values of (21) the $\mathrm{R}, \mathrm{B}$ update is completed using the proposed correlation-correction approach as follows:

$$
y_{0 k}= \begin{cases}y_{0 k} & \text { if } z_{0} \cong x_{0 k}, \\ y_{02}+\sum_{i=1}^{(N-1) / 2} w_{i}^{\prime \prime} & \text { if } z_{0} \cong x_{02}, \\ \quad \times\left(\bar{y}_{(2 i) k}-y_{(2 i) 2}\right) & \\ y_{02}+\sum_{i=1}^{(N-1) / 2} w_{i}^{\prime \prime \prime} & \\ \quad \times\left(\bar{y}_{(2 i-1) k}-y_{(2 i-1) 2}\right) & \text { if } z_{0} \cong x_{0(k \pm 2)} .\end{cases}
$$

Based on the simplicity of this step, in addition to the accuracy of the corrected $G$ values, the correction of the R,B values contributes significantly to the restored image fidelity.

Since $x_{0 k}$ of (1), $\bar{y}_{0 k}$ of (11) and (20); and $y_{0 k}$ of (21) and (22), for $z_{0} \cong x_{0 k}$ and $k=1,2,3$, are equivalent, the proposed ESCC method preserves all acquired (correct) values of (1) unchanged

$y_{i k}=x_{i k}$ for $z_{i} \cong x_{i k}$

leading to an efficient and attractive CFA interpolation method.

\subsection{Proposed modifications}

It was claimed in [31] that the color-difference model [18] is more appropriate for the image interpolation than the color-ratio model [8]. The reason is that the color-ratio model is based on the assumption of hue uniformity within a localized 
image area, and thus it fails near edge transitions where both the spectral and spatial correlation characteristics of the image vary significantly [32]. Moreover, the ratio calculations result in the singularities and outlying values which correspond to color artifacts in the images restored using the conventional color-ratio model based interpolators [32]. To overcome these limitations, the normalized color-ratio models have been successfully used in CFA interpolation [23] or demosaicked image postprocessing [32].

It is not difficult to see that due to the subtraction base the color-difference model is easier to implement and thus it is commonly used in numerous demosaicing solutions, e.g. [4,9-11,18]. However, there exists possibility that the color-difference model of (21) and (22) fails due to inadequate shifts in color caused by differences $\left(\bar{y}_{(2 i) 2}-\bar{y}_{(2 i) k}\right)$, $\left(\bar{y}_{(2 i) k}-y_{(2 i) 2}\right)$ and $\left(\bar{y}_{(2 i-1) k}-y_{(2 i-1) 2}\right)$. This can occur due to low correlation between the RGB channels and in the same time the correction process will decrease the image quality in terms of subjective and objective evaluation.

Because there is no reason to assume that low correlation between the R,G channels automatically denotes a similar relationship between the G,B channels, two different tests (for the R, G channels and G,B channels, respectively) are necessary.

Thus, the algorithm of the proposed edge-sensing global correlation-correction (GESCC) method (Fig. 7) can be stated as follows:

Input: $K_{1} \times K_{2}$ gray-scale image $z(l): Z^{2} \rightarrow Z$ Threshold parameter $\gamma$

Output: $K_{1} \times K_{2}$ color image $\mathbf{y}(l): Z^{2} \rightarrow Z^{3}$

Create $K_{1} \times K_{2}$ RGB image $\mathbf{x}(l): Z^{2} \rightarrow Z^{3}$ using (1)

Edge-detection process

Count edge-sensing coefficients $w_{1}, w_{2}, \ldots, w_{8}$

defined by (3) to (10)

Determine normalized weights

$w_{i}^{\prime}=w_{i} / \sum_{j=1}^{N-1} w_{j}$, for $i=1,2, \ldots, N-1$

Count assistance values $x_{(1) 2}^{\prime}, x_{(2) 2}^{\prime}, \ldots, x_{(8) 2}^{\prime}$ of

(12) to (19)

Interpolation process

Determine the input set $W=\mathbf{x}_{0}, \mathbf{x}_{1}, \ldots, \mathbf{x}_{8}$ of

$N=9$ samples

Let the $G$ value according to (11)

Determine normalized weights

$w_{i}^{\prime \prime}=w_{2 i} / \sum_{j=1}^{(N-1) / 2} w_{2 j}$, for $i=1,2, \ldots, 4$
Determine normalized weights

$w_{i}^{\prime \prime \prime}=w_{(2 i-1)} / \sum_{j=1}^{(N-1) / 2} w_{(2 j-1)}$, for $i=1,2, \ldots, 4$

Let the R,B values according to (20)

High correlation detection

Perform high-pass filtering of $x_{i 1}, x_{i 3}$ and $\bar{y}_{i 2}$

Let correlation coefficients $C_{1}$ and $C_{3}$ according

to (24), see Fig. 10

Correction process

If $C_{1}>\gamma$ and $C_{3}>\gamma$

Let the $\mathrm{G}$ values according to (21)

If $C_{1}>\gamma$

Let the $\mathrm{R}$ value according to (22)

If $C_{3}>\gamma$

Let the B value according to (22)

Output $K_{1} \times K_{2}$ restored RGB image

$\mathbf{y}(l): Z^{2} \rightarrow Z^{3}$

Comparing the ESCC algorithm and the GESCC method, a novel part introduces a global switching mode between the non-correction of the R,B values and their correction depending on global parameters, correlation coefficients, given by

$C_{k}=\frac{\sum\left(\tilde{x}_{i k}-\hat{x}_{k}\right)\left(\tilde{y}_{i 2}-\hat{y}_{2}\right)}{\sqrt{\sum\left(\tilde{x}_{i k}-\hat{x}_{k}\right)^{2}} \sqrt{\sum\left(\tilde{y}_{i 2}-\hat{y}_{2}\right)^{2}}}$ for $z_{i} \cong x_{i k}$,

where $i$ characterizes the spatial position of the samples corresponding to the original position of $\mathrm{R}, \mathrm{B}$ values in the Bayer pattern, $\hat{x}_{k}$ denotes the sample mean related to the $k$ th channel values of $\tilde{\mathbf{x}}(l)$ and $\hat{y}_{2}$ is the sample mean related to the $\mathrm{G}$ channel of $\overline{\mathbf{y}}(l)$ defined as follows:

$\tilde{x}_{i k}=f_{1,-2,1}\left(x_{(i-1) k}, x_{i k}, x_{(i+1) k}\right)$ for $\forall\left(z_{(\cdot)} \cong x_{(\cdot) k}\right)$,

$\tilde{y}_{i 2}=f_{1,-2,1}\left(\bar{y}_{(i-1) 2}, \bar{y}_{i 2}, \bar{y}_{(i+1) 2}\right)$ for $\forall\left(z_{(\cdot)} \cong x_{(\cdot) k}\right)$,

where $f_{1,-2,1}(\cdot)$ denotes a 1-D high pass filtering (Fig. 8) with the coefficients $(1,-2,1)$ applied to horizontal and vertical directions, $k$ characterizes the $\mathrm{R}(k=1)$ and $\mathrm{B}(k=3)$ channels.

If $C_{k}$ is larger than a chosen threshold $\gamma$, then (22) carries out for the appropriate color channel $k$. Otherwise, the corresponding channel is not corrected. If the correction process is completely 
omitted the GESCC solution reduces to the C2D2 method. Thus, in the images with very weak correlation characteristics, the improvement of the GESCC method compared to the basic ESCC case developed within the proposed framework is always limited to the performance of the C2D2 solution.

However, situations may occur when it is desirable to switch between the correction mode and the interpolation process depending on the local image statistics. The methods developed in the past were designed to follow strong correlation between all color channels and strong correlation between either R,G or G,B channels. Methods appropriate for images with a weak correlation have been developed as well. However, natural images typically consist of alternating regions of high and low correlation between the color channels. Therefore, we introduce another method which takes into account varied correlation of image areas.

The proposed edge-sensing local correlation-correction (LESCC) approach (Fig. 9) performs the

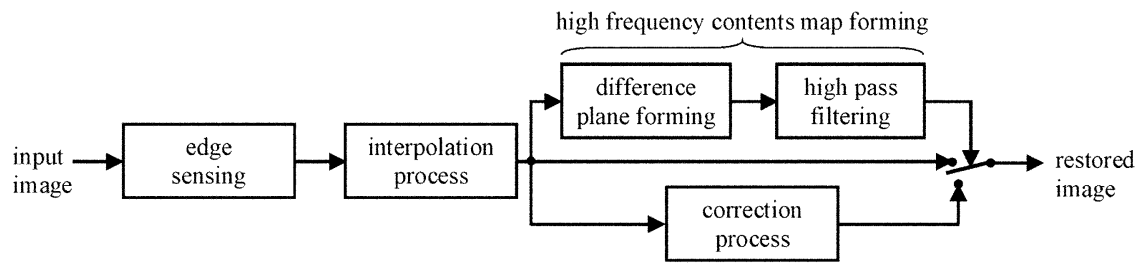

Fig. 7. Block scheme of the proposed GESCC method.

(a)

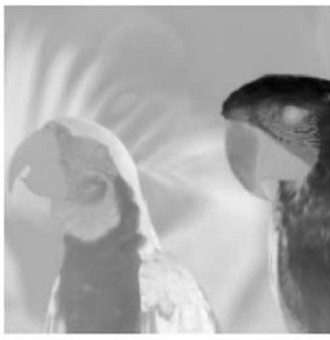

(e)

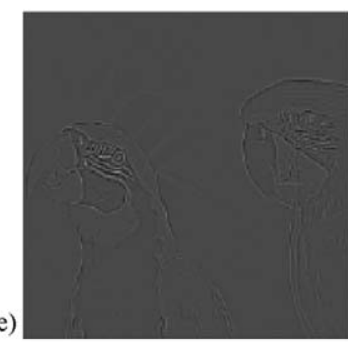

(b)

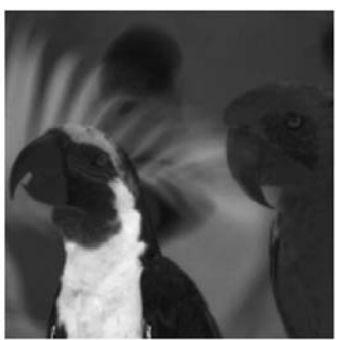

(f)

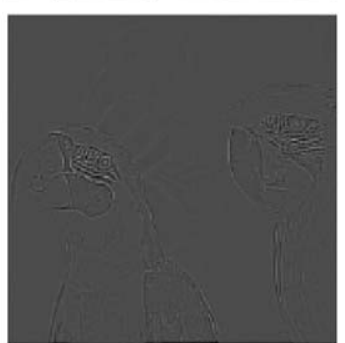

(c)

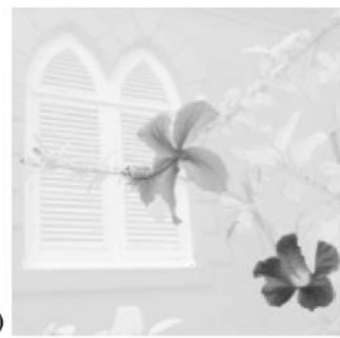

(g)

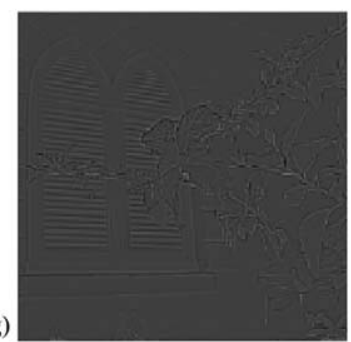

(d)

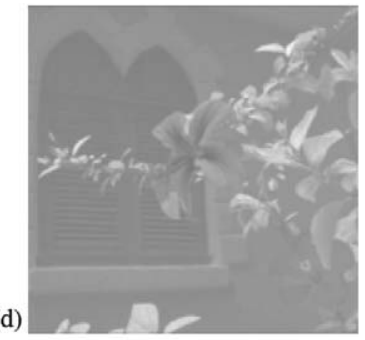

(h)

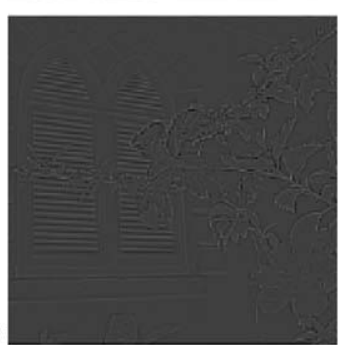

Fig. 8. Forming of the difference planes and the high frequency content maps: (a) R-G and (b) G-B difference plane of the image Parrots, (c) $\mathrm{R}-\mathrm{G}$ and (d) $\mathrm{G}-\mathrm{B}$ difference plane of the image window; (e-h) high frequency maps formed by a high pass filtering of the corresponding difference planes shown in $(\mathrm{a}-\mathrm{d})$.

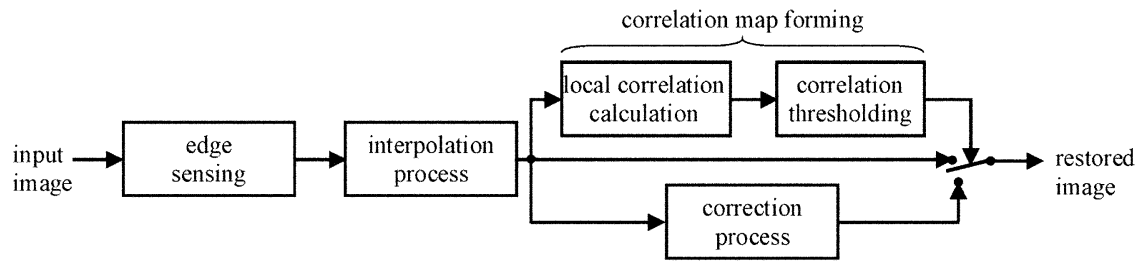

Fig. 9. Block scheme of the proposed LESCC method. 
(a)

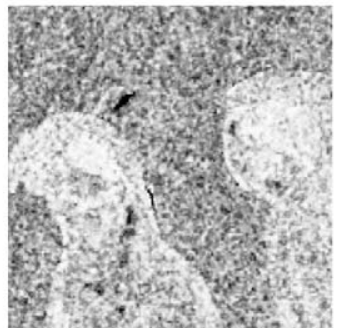

(e)

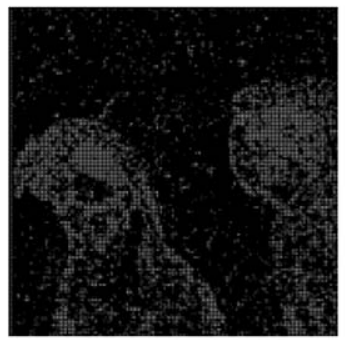

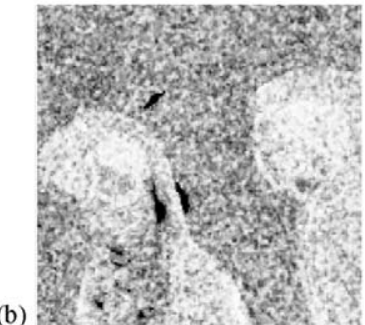

(b)

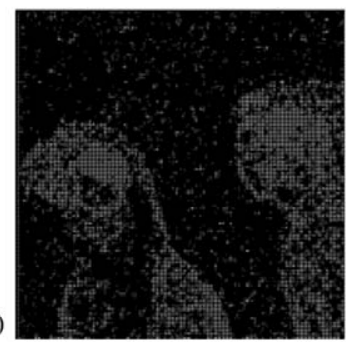

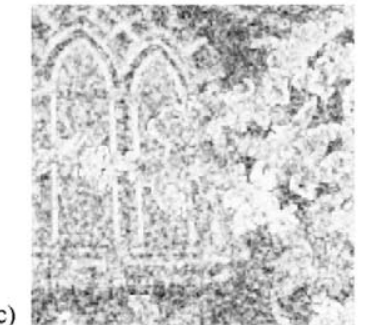

(c)

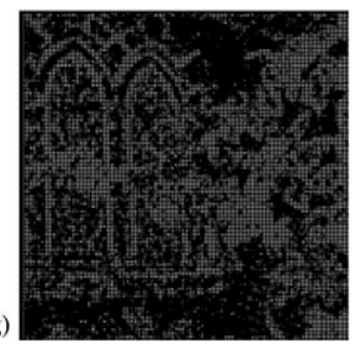

(d)

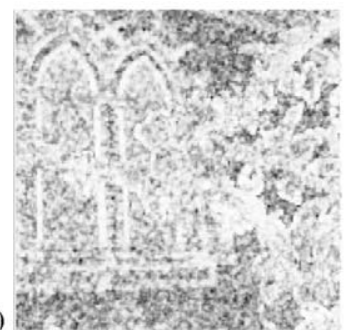

(h)

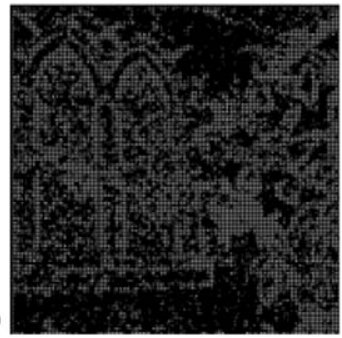

Fig. 10. Forming of the correlation maps: (a) R-G and (b) G-B local correlation map of the image Parrots, (c) R-G and (d) G-B local correlation map of the image window; $(\mathrm{e}-\mathrm{h})$ correlation maps formed by a thresholding of the corresponding local correlation maps shown in $(\mathrm{a}-\mathrm{d})$.

(a)

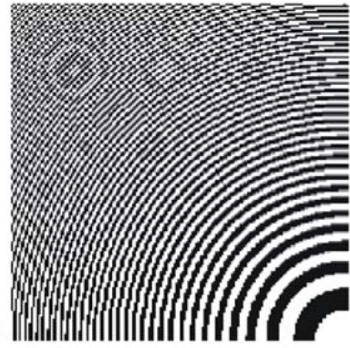

(e)

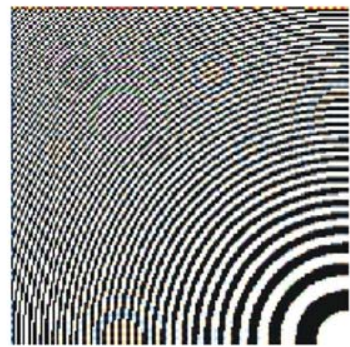

(b)
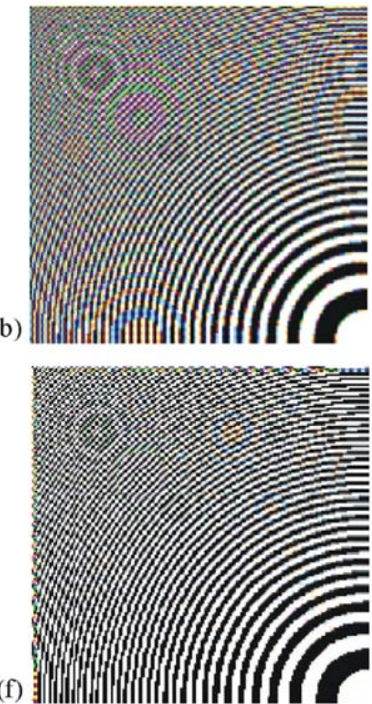
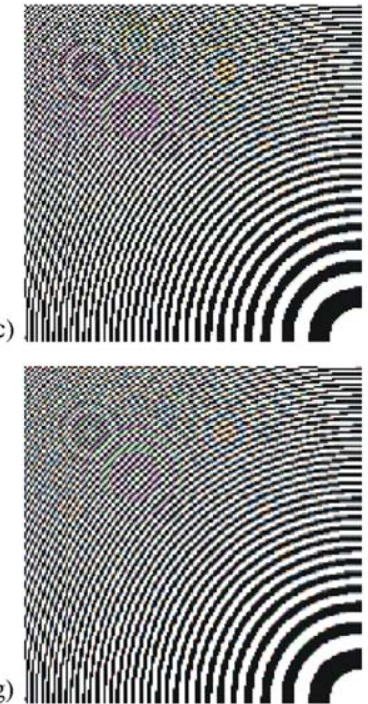

(d)
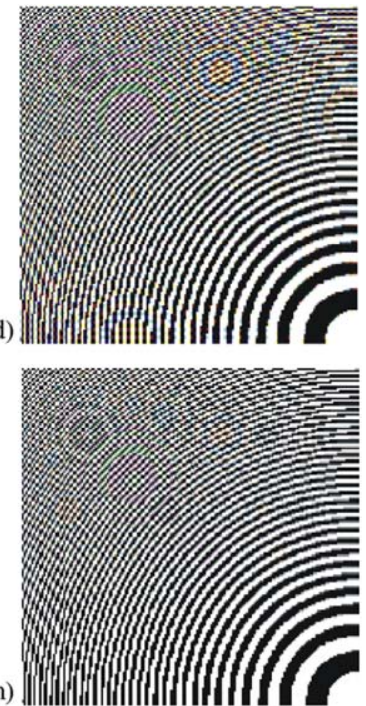

Fig. 11. Top-left patterns of the results related to the Circular Zone Plate (CZP) image: (a) original image, (b) BI, (c) DCCA, (d) MFI, (e) $\mathrm{BD}$, (f) $\mathrm{AP},(\mathrm{g}) \mathrm{C} 2 \mathrm{D} 2$, (h) proposed ESCC scheme.

identical edge-sensing and interpolation steps as the GESCC method. However, the LESCC method utilizes correlation maps (Fig. 10) between the interpolated $\mathrm{G}$ channel of $\overline{\mathbf{y}}(l)$ and the original $\mathrm{R}, \mathrm{B}$ channels of $\mathbf{x}(l)$, for $\forall\left(z_{(\cdot)} \cong x_{(\cdot) k}\right)$. Note that these correlation maps are calculated using $3 \times 3$ neighborhoods. Based on the input parameter $\beta$, correction operations are performed for the local correlation larger than $\beta$.

\section{Experimental results}

To examine the performance of the proposed framework and facilitate comparisons with the state of-the-art CFA interpolation schemes reviewed in this paper, some widely used test images, such as the Circular Zone Plate (CZP) image of Fig. 11a and the natural color images of the database shown in Fig. 12 are utilized. All test images have been 

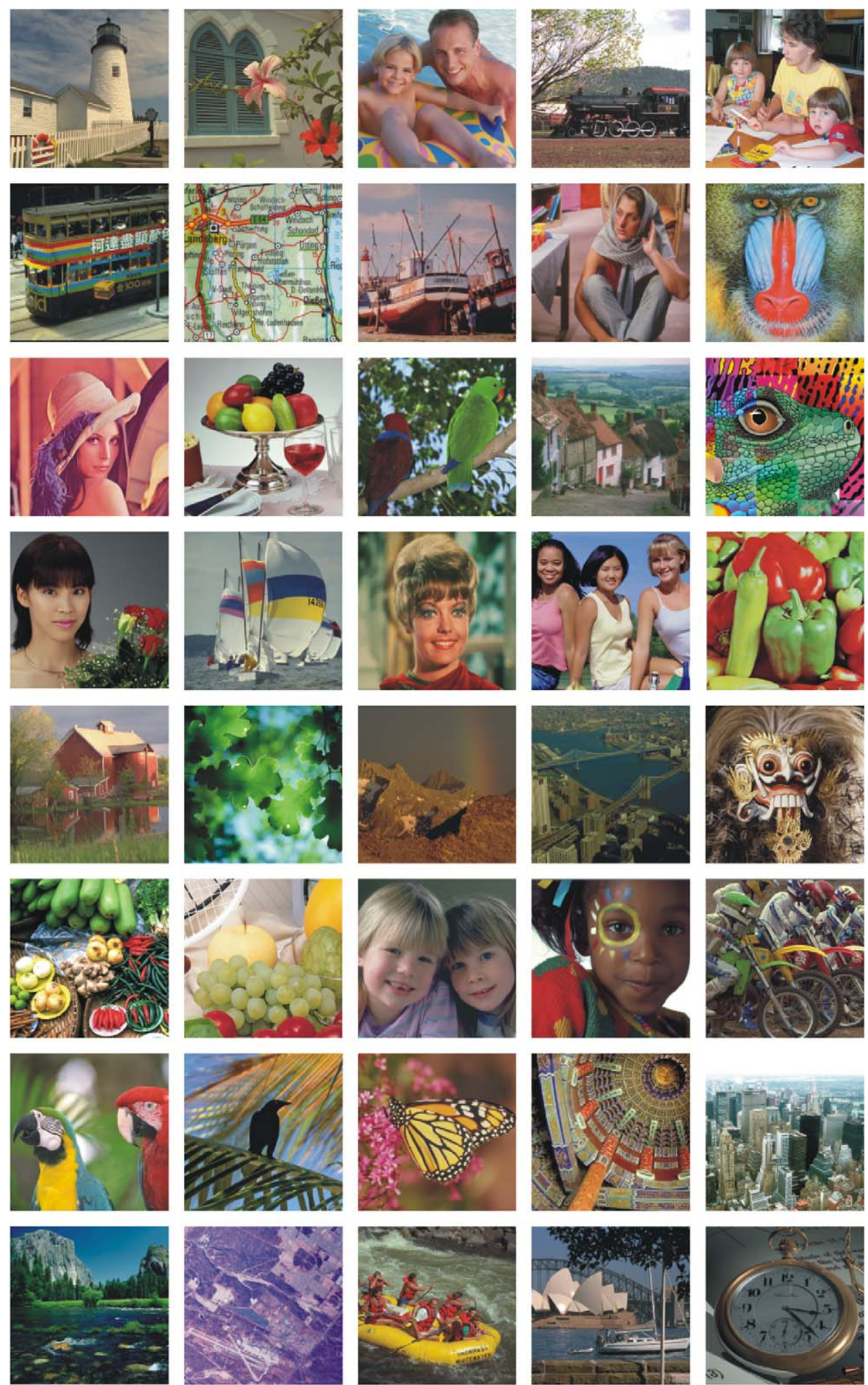

Fig. 12. Used test image database. 
normalized to a standard size of $512 \times 512$ pixels with a 8-bit per channel RGB representation.

Methods (i.e. ESCC, GESCC, LESCC) constructed within the proposed framework are compared, in terms of performance, with the CFA interpolation methods listed in Table 1. Following common practice in the research community, mosaic versions of the images are created by discarding color information in a GRGR phased Bayer CFA filter [6,10,32]. Demosaicked images are generated using each of the listed methods. To measure similarity between the original RGB image $\mathbf{o}(l)$ and interpolated (restored) image $\mathbf{y}(l)$, the mean square error (MSE) and the peak signal to noise ratio (PSNR) are used to evaluate objectively the quality of the restored RGB channels.

The MSE and PSNR criteria are defined as follows:

$\operatorname{MSE}_{k}=\frac{1}{K_{1} K_{2}} \sum_{l=1}^{K_{1} K_{2}}\left(o_{l k}-y_{l k}\right)^{2}$,

$\operatorname{PSNR}_{k}=20 \log _{10}\left(255 / \sqrt{\mathrm{MSE}_{k}}\right)$,

where $\mathbf{o}_{l}=\left(o_{l 1}, o_{l 2}, o_{l 3}\right)$ is the original pixel, $\mathbf{y}_{l}=$ $\left(y_{l 1}, y_{l 2}, y_{l 3}\right)$ is the restored pixel, $l$ is the pixel position in a $K_{1} \times K_{2}$ color image and $k$ characterizes the color channel.

To quantify the perceptual closeness between the original and the obtained solution, the normalized

Table 1

Methods taken for comparison with the proposed interpolation framework (i.e. ESCC, GESCC, LESCC)

\begin{tabular}{lll}
\hline Notation & Method & Ref. \\
\hline NNI & Nearest neighbor interpolation & {$[20,33]$} \\
BI & Bilinear interpolation & {$[22,21]$} \\
BCI & Bicubic interpolation & {$[34]$} \\
SHT & Smooth hue transition approach & {$[8]$} \\
VMI & Vector median interpolation & {$[19]$} \\
SVF & Spatial varying filtering & {$[16]$} \\
DCCA & Discriminated color correlation approach & {$[31]$} \\
MFI & Median filter interpolation & {$[9]$} \\
API & Adaptive color plane interpolation & {$[11]$} \\
BD & Bilinear difference interpolation & {$[10]$} \\
TDA & Triangulation difference approach & {$[35]$} \\
PVM & Principle vector method & {$[12]$} \\
AP & Alternating projection approach & {$[6]$} \\
KA & Kimmel algorithm & {$[13]$} \\
C2D2 & Color correlation-directional derivatives & {$[17]$} \\
SAIG & Saturation based adaptive inverse gradient & {$[27]$} \\
EMI & Edge map interpolation & {$[28]$} \\
LIM & Layering interpolation approach & {$[30]$} \\
\hline
\end{tabular}

difference criterion (NCD) of [2] is used in this work. The NCD is given by

$\mathrm{NCD}$

$$
=\frac{\sum_{l=1}^{K_{1} K_{2}} \sqrt{\left(L_{\mathbf{o}_{l}}^{*}-L_{\mathbf{y}_{l}}^{*}\right)^{2}+\left(u_{\mathbf{o}_{l}}^{*}-u_{\mathbf{y}_{l}}^{*}\right)^{2}+\left(v_{\mathbf{o}_{l}}^{*}-v_{\mathbf{y}_{l}}^{*}\right)^{2}}}{\sum_{l=1}^{K_{1} K_{2}} \sqrt{\left(L_{\mathbf{o}_{l}}^{*}\right)^{2}+\left(u_{\mathbf{o}_{l}}^{*}\right)^{2}+\left(v_{\mathbf{o}_{l}}^{*}\right)^{2}}},
$$

where $L^{*}$ represents lightness values and $\left(u^{*}, v^{*}\right)$ chrominance values corresponding to original $\mathbf{o}_{l}$ and restored $\mathbf{y}_{l}$ samples expressed in CIELUV color space [3] with the white point D65.

Table 2 summarizes results obtained using the artificial CZP image [10] shown in Fig. 11a. Note that the CZP image is a black and white image commonly used to demonstrate the degree of zipper effect and aliasing artifacts present in the restored image $[17,10]$. Since the frequency of the edges increases in vertical, horizontal and diagonal directions, the lack of interpolation accuracy caused by the nature (or omission) of the edge-sensing mechanism, spectral (color) model, correction step, or a combination of these can be easily observed and precisely evaluated. These results and the corresponding restored images depicted in Figs. $11 \mathrm{~b}-\mathrm{h}$ clearly demonstrate that the proposed framework is capable of restoring the CZP image with the highest accuracy comparing to the state of-the-art CFA interpolation methods reviewed here. It is evident that edge-sensing methods outperform non-adaptive interpolation techniques. The performance of the C2D2 method and the ESCC framework with their eight-directional edge-sensing mechanism demonstrate that these methods are capable of preserving the high frequency image contents in all directions. Moreover, the edge-sensing correction step introduced by the proposed framework results in the best performance among the methods compared.

Fig. 13 illustrates the sensitivity of proposed GESCC and LESCC methods to the design parameter $\gamma$ and $\beta$, respectively. As it can be observed, values around $\gamma=0.450$ and $\beta=0.125$ deliver excellent results and the trade-off between the error criteria (PSNR or MSE vs. NCD). Note that these values are globally optimal for the image database shown in Fig. 12 and are used in the rest of this paper.

Tables 3-6 summarize results corresponding to the restoration of some well-known test images (Lighthouse, Window, Water, Train) shown in Fig. 12. Using natural test images we are able to compare performance of the methods in realistic 
Table 2

Comparison of the methods using the CZP test image

\begin{tabular}{|c|c|c|c|c|c|c|c|}
\hline Method & $\mathrm{MSE}_{\mathrm{R}}$ & $\mathrm{MSE}_{\mathrm{G}}$ & $\mathrm{MSE}_{\mathrm{B}}$ & $\mathrm{PSNR}_{\mathrm{R}}$ & $\mathrm{PSNR}_{\mathrm{G}}$ & $\mathrm{PSNR}_{\mathrm{B}}$ & $\mathrm{NCD}$ \\
\hline NNI & 9367.72 & 3300.70 & 9342.85 & 8.41 & 12.94 & 8.42 & 1.0062 \\
\hline BI & 3804.12 & 1775.17 & 3849.09 & 12.32 & 15.63 & 12.27 & 0.8433 \\
\hline BCI & 3119.59 & 1418.79 & 3154.91 & 13.19 & 16.61 & 13.14 & 0.7706 \\
\hline SHT & 1872.90 & 1775.17 & 1903.99 & 15.40 & 15.63 & 15.33 & 0.4832 \\
\hline VMI & 9542.26 & 7126.87 & 9442.66 & 8.33 & 9.60 & 8.38 & 0.9401 \\
\hline SVF & 1506.08 & 337.51 & 1496.74 & 16.35 & 22.84 & 16.37 & 0.5077 \\
\hline DCCA & 269.17 & 180.44 & 255.54 & 23.83 & 25.56 & 24.05 & 0.1172 \\
\hline MFI & 984.47 & 135.13 & 997.33 & 18.19 & 26.82 & 18.14 & 0.4332 \\
\hline API & 910.79 & 131.66 & 926.17 & 18.53 & 26.93 & 18.46 & 0.4891 \\
\hline $\mathrm{BD}$ & 408.78 & 180.29 & 413.30 & 22.01 & 25.57 & 21.96 & 0.4237 \\
\hline TDA & 221.28 & 114.18 & 221.08 & 24.68 & 27.55 & 24.68 & 0.3075 \\
\hline PVM & 875.06 & 1361.68 & 884.76 & 18.71 & 16.79 & 18.66 & 0.4173 \\
\hline AP & 547.85 & 646.92 & 545.48 & 20.74 & 20.02 & 20.76 & 0.4348 \\
\hline KA & 1053.02 & 937.71 & 1077.78 & 17.90 & 18.41 & 17.80 & 0.3609 \\
\hline C2D2 & 319.33 & 553.62 & 326.10 & 23.08 & 20.69 & 22.99 & 0.3440 \\
\hline SAIG & 1377.45 & 413.74 & 1403.37 & 16.74 & 21.96 & 16.65 & 0.5834 \\
\hline EMI & 970.77 & 319.45 & 916.57 & 18.26 & 23.08 & 18.50 & 0.3682 \\
\hline LIM & 1339.88 & 669.89 & 1345.84 & 16.86 & 19.87 & 16.84 & 0.3640 \\
\hline ESCC & 78.06 & 78.58 & 78.05 & 29.20 & 29.17 & 29.20 & 0.2281 \\
\hline GESCC & 78.06 & 78.58 & 78.05 & 29.20 & 29.17 & 29.20 & 0.2281 \\
\hline LESCC & 78.06 & 78.58 & 78.05 & 29.20 & 29.17 & 29.20 & 0.2281 \\
\hline
\end{tabular}

applications, since in these images the correlation between the color channels vary significantly. As the reported error values indicate, flexible design characteristics of the proposed method result in excellent performance.

Figs. 14 and 15 present zoomed parts of the restored images. These images allow for the comparison of the state of-the-art CFA interpolation results with those obtained through our framework in terms of a subjective (user-centered) evaluation. It is evident that many interpolation methods (i) fail near edges and (ii) produce color artifacts. Most methods blur edges and fine details as a result of the inefficient edge-sensing mechanism. However, the proposed framework is capable of restoring the color images with a high visual quality and avoids color shifts and visual artifacts. Therefore, the proposed framework produces impressive visual quality of the restored images shown in Fig. $15 \mathrm{j}$, which is also demonstrated by their superior fidelity in comparison with the original patterns shown in Fig. 14a.

Figs. 16 and 17 list estimation errors obtained using the most powerful CFA interpolation techniques. It can be seen that many methods are characterized by a significant restoration inaccuracy caused by edge blurring. Some of the methods increase estimation error introducing color artifacts.
The proposed framework, in addition to color chrominance preservation capability, maintains excellent signal-detail preserving characteristics, resulting in the smallest estimation error depicted in Fig. 17e.

To demonstrate the robustness of the method, Table 7 presents results corresponding to the image database shown in Fig. 12. These numerical values were achieved applying the CFA methods for all 40 test images and then averaging the aggregated error criteria. The proposed framework exhibits the best performance and achieves an excellent improvement in comparison with previously developed approaches.

Apart from the actual performance of any algorithm, its computational complexity is a realistic measure of its practicality and usefulness. Table 8 lists the average computational time per a $512 \times 512$ input image noticed for the proposed framework as well as some other sophisticated CFA interpolation solutions. The execution of the developed postprocessing tool was performed on a personal computer with an Intel Pentium IV $2.53 \mathrm{GHz}$ CPU, $512 \mathrm{MB}$ RAM, Red Hat Linux 9 operating system and Matlab 6.5 programming environment. As it can be seen, all solutions (ESCC, GESCC, LESCC) designed within the proposed framework belong with the C2D2 method to the 

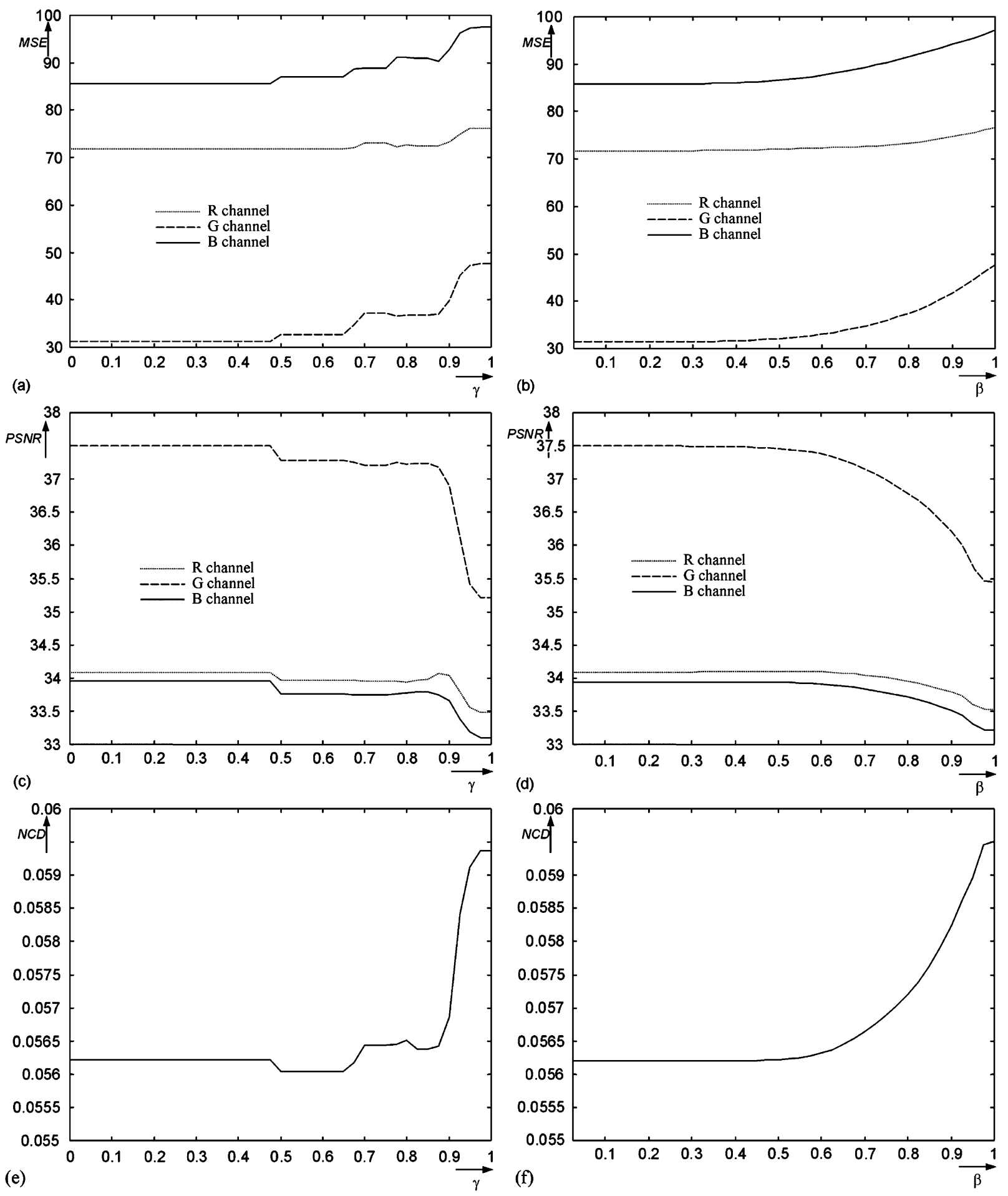

Fig. 13. Performance of the proposed GESCC (a,c,e) and LESCC (b,d,f) methods dependent on the threshold value $\gamma$ and $\beta$, respectively. These results corresponding to the image database shown in Fig. 12 were achieved as aggregated error measures averaged through the number of the test images: (a,b) MSE criteria, (c,d) PSNR criteria, (e,f) NCD criteria. 
Table 3

Comparison of the methods using the test image Lighthouse

\begin{tabular}{|c|c|c|c|c|c|c|c|}
\hline Method & $\mathrm{MSE}_{\mathrm{R}}$ & $\mathrm{MSE}_{\mathrm{G}}$ & $\mathrm{MSE}_{\mathrm{B}}$ & $\mathrm{PSNR}_{\mathrm{R}}$ & $\mathrm{PSNR}_{\mathrm{G}}$ & $\mathrm{PSNR}_{\mathrm{B}}$ & NCD \\
\hline NNI & 280.97 & 81.91 & 265.32 & 23.64 & 28.99 & 23.89 & 0.0927 \\
\hline BI & 138.69 & 44.32 & 134.23 & 26.71 & 31.66 & 26.85 & 0.0653 \\
\hline $\mathrm{BCI}$ & 138.62 & 42.95 & 137.01 & 26.71 & 31.80 & 26.76 & 0.0679 \\
\hline SHT & 57.64 & 44.32 & 66.29 & 30.52 & 31.66 & 29.91 & 0.0501 \\
\hline VMI & 214.66 & 113.91 & 192.94 & 24.81 & 27.56 & 25.27 & 0.0753 \\
\hline SVF & 131.33 & 32.90 & 127.51 & 26.94 & 32.95 & 27.07 & 0.0631 \\
\hline DCCA & 27.74 & 12.53 & 19.58 & 33.69 & 37.15 & 35.21 & 0.0361 \\
\hline MFI & 44.28 & 7.94 & 40.78 & 31.66 & 39.13 & 32.02 & 0.0380 \\
\hline API & 14.57 & 9.71 & 13.19 & 36.49 & 38.25 & 36.92 & 0.0298 \\
\hline $\mathrm{BD}$ & 23.24 & 12.65 & 21.91 & 34.46 & 37.10 & 34.72 & 0.0321 \\
\hline TDA & 40.15 & 21.64 & 46.64 & 32.09 & 34.77 & 31.44 & 0.0418 \\
\hline PVM & 23.92 & 21.83 & 23.60 & 34.34 & 34.74 & 34.40 & 0.0366 \\
\hline $\mathrm{AP}$ & 9.15 & 4.74 & 7.40 & 38.51 & 41.36 & 39.43 & 0.0237 \\
\hline KA & 16.97 & 16.38 & 26.35 & 35.83 & 35.98 & 33.92 & 0.0317 \\
\hline $\mathrm{C} 2 \mathrm{D} 2$ & 16.94 & 13.16 & 17.57 & 35.84 & 36.93 & 35.68 & 0.0301 \\
\hline SAIG & 109.40 & 16.71 & 107.04 & 27.74 & 35.90 & 27.83 & 0.0588 \\
\hline EMI & 21.83 & 16.36 & 29.56 & 34.74 & 35.99 & 33.42 & 0.0370 \\
\hline LIM & 22.70 & 16.83 & 24.12 & 34.57 & 35.87 & 34.30 & 0.0363 \\
\hline ESCC & 10.30 & 5.25 & 9.95 & 38.00 & 40.92 & 38.15 & 0.0236 \\
\hline GESCC & 10.30 & 5.25 & 9.95 & 38.00 & 40.92 & 38.15 & 0.0236 \\
\hline LESCC & 10.30 & 5.25 & 9.95 & 38.00 & 40.92 & 38.15 & 0.0236 \\
\hline
\end{tabular}

Table 4

Comparison of the methods using the test image Window

\begin{tabular}{|c|c|c|c|c|c|c|c|}
\hline Method & $\mathrm{MSE}_{\mathrm{R}}$ & $\mathrm{MSE}_{\mathrm{G}}$ & $\mathrm{MSE}_{\mathrm{B}}$ & $\mathrm{PSNR}_{\mathrm{R}}$ & $\mathrm{PSNR}_{\mathrm{G}}$ & $\mathrm{PSNR}_{\mathrm{B}}$ & NCD \\
\hline NNI & 123.66 & 41.63 & 127.62 & 27.20 & 31.93 & 27.07 & 0.0747 \\
\hline BI & 41.48 & 17.24 & 41.61 & 31.95 & 35.76 & 31.93 & 0.0417 \\
\hline $\mathrm{BCI}$ & 36.44 & 14.50 & 36.43 & 32.51 & 36.51 & 32.51 & 0.0372 \\
\hline SHT & 21.25 & 17.24 & 20.74 & 34.85 & 35.76 & 34.96 & 0.0329 \\
\hline VMI & 95.82 & 65.76 & 100.71 & 28.31 & 29.95 & 28.10 & 0.0633 \\
\hline SVF & 41.23 & 13.27 & 41.37 & 31.97 & 36.90 & 31.96 & 0.0411 \\
\hline DCCA & 37.84 & 19.92 & 16.71 & 32.35 & 35.13 & 35.90 & 0.0342 \\
\hline MFI & 9.50 & 2.88 & 9.78 & 38.35 & 43.53 & 38.22 & 0.0239 \\
\hline API & 7.03 & 4.53 & 7.11 & 39.65 & 41.56 & 39.608 & 0.0209 \\
\hline $\mathrm{BD}$ & 7.06 & 4.57 & 7.92 & 39.64 & 41.52 & 39.14 & 0.0228 \\
\hline TDA & 10.79 & 6.90 & 15.92 & 37.79 & 39.74 & 36.11 & 0.0306 \\
\hline PVM & 11.87 & 11.95 & 12.53 & 37.38 & 37.35 & 37.15 & 0.0247 \\
\hline $\mathrm{AP}$ & 6.16 & 3.38 & 6.61 & 40.23 & 42.84 & 39.92 & 0.0212 \\
\hline KA & 11.69 & 10.10 & 12.59 & 37.45 & 38.08 & 37.13 & 0.0256 \\
\hline $\mathrm{C} 2 \mathrm{D} 2$ & 6.19 & 5.67 & 6.70 & 40.21 & 40.58 & 39.86 & 0.0193 \\
\hline SAIG & 23.95 & 7.57 & 23.50 & 34.33 & 39.33 & 34.41 & 0.0322 \\
\hline EMI & 12.42 & 9.80 & 11.33 & 37.18 & 38.21 & 37.58 & 0.0252 \\
\hline LIM & 17.40 & 6.98 & 18.10 & 35.72 & 39.69 & 35.55 & 0.0318 \\
\hline ESCC & 4.88 & 2.27 & 5.52 & 41.24 & 44.55 & 40.70 & 0.0181 \\
\hline GESCC & 4.88 & 2.27 & 5.52 & 41.24 & 44.55 & 40.70 & 0.0181 \\
\hline LESCC & 4.88 & 2.27 & 5.52 & 41.24 & 44.55 & 40.70 & 0.0182 \\
\hline
\end{tabular}


Table 5

Comparison of the methods using the test image Water

\begin{tabular}{|c|c|c|c|c|c|c|c|}
\hline Method & $\mathrm{MSE}_{\mathrm{R}}$ & $\mathrm{MSE}_{\mathrm{G}}$ & $\mathrm{MSE}_{\mathrm{B}}$ & $\mathrm{PSNR}_{\mathrm{R}}$ & $\mathrm{PSNR}_{\mathrm{G}}$ & $\mathrm{PSNR}_{\mathrm{B}}$ & NCD \\
\hline NNI & 50.01 & 8.87 & 45.54 & 31.14 & 38.65 & 31.54 & 0.0356 \\
\hline $\mathrm{BI}$ & 13.23 & 3.56 & 12.34 & 36.91 & 42.60 & 37.21 & 0.0194 \\
\hline $\mathrm{BCI}$ & 10.49 & 2.90 & 10.47 & 37.92 & 43.50 & 37.93 & 0.0184 \\
\hline SHT & 9.90 & 3.56 & 9.17 & 38.17 & 42.60 & 38.50 & 0.0194 \\
\hline VMI & 39.54 & 15.45 & 34.54 & 32.16 & 36.24 & 32.74 & 0.0312 \\
\hline SVF & 14.76 & 2.98 & 13.54 & 36.44 & 43.38 & 36.81 & 0.0205 \\
\hline DCCA & 35.08 & 35.70 & 97.87 & 32.68 & 32.60 & 28.22 & 0.0304 \\
\hline MFI & 8.31 & 2.76 & 7.73 & 38.93 & 43.71 & 39.24 & 0.0189 \\
\hline API & 8.84 & 2.31 & 8.79 & 38.66 & 44.49 & 38.68 & 0.0193 \\
\hline $\mathrm{BD}$ & 11.38 & 3.50 & 13.32 & 37.56 & 42.68 & 36.88 & 0.0212 \\
\hline TDA & 17.71 & 6.10 & 20.52 & 35.64 & 40.27 & 35.00 & 0.0250 \\
\hline PVM & 6.60 & 2.33 & 7.77 & 39.93 & 44.45 & 39.22 & 0.0177 \\
\hline $\mathrm{AP}$ & 15.60 & 7.80 & 17.40 & 36.19 & 39.20 & 35.72 & 0.0255 \\
\hline KA & 7.80 & 2.04 & 6.70 & 39.2 & 45.02 & 39.86 & 0.0182 \\
\hline $\mathrm{C} 2 \mathrm{D} 2$ & 6.40 & 1.63 & 6.83 & 40.06 & 45.99 & 39.78 & 0.0177 \\
\hline SAIG & 9.33 & 1.86 & 9.47 & 38.43 & 45.42 & 38.36 & 0.0186 \\
\hline EMI & 9.53 & 2.66 & 7.75 & 38.33 & 43.87 & 39.23 & 0.0191 \\
\hline LIM & 27.18 & 8.84 & 28.92 & 33.78 & 38.66 & 33.51 & 0.0256 \\
\hline ESCC & 8.983 & 3.11 & 9.48 & 38.59 & 43.20 & 38.35 & 0.0215 \\
\hline GESCC & 6.40 & 1.63 & 6.83 & 40.06 & 45.99 & 39.78 & 0.0177 \\
\hline LESCC & 7.19 & 1.80 & 7.22 & 39.55 & 45.57 & 39.54 & 0.0183 \\
\hline
\end{tabular}

Table 6

Comparison of the methods using the test image Train

\begin{tabular}{|c|c|c|c|c|c|c|c|}
\hline Method & $\mathrm{MSE}_{\mathrm{R}}$ & $\mathrm{MSE}_{\mathrm{G}}$ & $\mathrm{MSE}_{\mathrm{B}}$ & $\mathrm{PSNR}_{\mathrm{R}}$ & $\mathrm{PSNR}_{\mathrm{G}}$ & $\mathrm{PSNR}_{\mathrm{B}}$ & NCD \\
\hline NNI & 1119.35 & 449.15 & 1345.11 & 17.64 & 21.60 & 16.84 & 0.2080 \\
\hline BI & 517.71 & 254.59 & 607.19 & 20.99 & 24.07 & 20.29 & 0.1450 \\
\hline $\mathrm{BCI}$ & 522.68 & 244.53 & 606.38 & 20.94 & 24.24 & 20.30 & 0.1513 \\
\hline SHT & 220.32 & 254.59 & 282.44 & 24.70 & 24.07 & 23.62 & 0.1063 \\
\hline VMI & 911.56 & 792.60 & 1052.45 & 18.53 & 19.14 & 17.90 & 0.1693 \\
\hline SVF & 450.91 & 188.44 & 531.90 & 21.59 & 25.37 & 20.87 & 0.1345 \\
\hline DCCA & 93.75 & 62.44 & 123.54 & 28.41 & 30.17 & 27.21 & 0.0733 \\
\hline MFI & 115.44 & 46.03 & 163.34 & 27.50 & 31.50 & 26.00 & 0.0798 \\
\hline API & 109.24 & 96.62 & 145.01 & 27.74 & 28.28 & 26.51 & 0.0776 \\
\hline BD & 74.71 & 65.45 & 113.47 & 29.39 & 29.97 & 27.58 & 0.0744 \\
\hline TDA & 86.32 & 69.26 & 147.30 & 28.77 & 29.72 & 26.44 & 0.0782 \\
\hline PVM & 187.46 & 220.33 & 230.24 & 25.40 & 24.70 & 24.50 & 0.0958 \\
\hline AP & 44.43 & 30.80 & 78.39 & 31.65 & 33.24 & 29.18 & 0.0560 \\
\hline KA & 71.22 & 85.68 & 138.41 & 29.60 & 28.80 & 26.71 & 0.0641 \\
\hline $\mathrm{C} 2 \mathrm{D} 2$ & 93.30 & 113.51 & 128.97 & 28.43 & 27.58 & 27.02 & 0.0702 \\
\hline SAIG & 387.31 & 162.39 & 456.27 & 22.25 & 26.02 & 21.53 & 0.1246 \\
\hline EMI & 159.42 & 152.53 & 195.69 & 26.10 & 26.29 & 25.21 & 0.0845 \\
\hline LIM & 175.69 & 66.42 & 223.59 & 25.68 & 29.90 & 24.63 & 0.0910 \\
\hline ESCC & 40.44 & 31.24 & 71.69 & 32.06 & 33.18 & 29.57 & 0.0503 \\
\hline GESCC & 40.44 & 31.24 & 71.69 & 32.06 & 33.18 & 29.57 & 0.0503 \\
\hline LESCC & 40.43 & 31.24 & 71.65 & 32.06 & 33.18 & 29.57 & 0.0503 \\
\hline
\end{tabular}



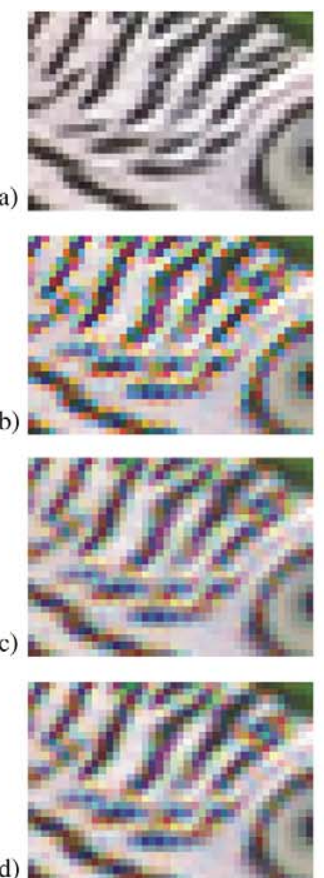

(d) $t$

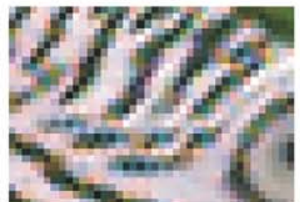

(e)

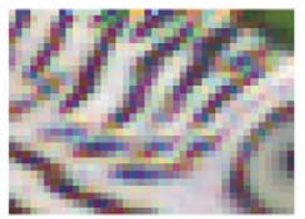

(g)
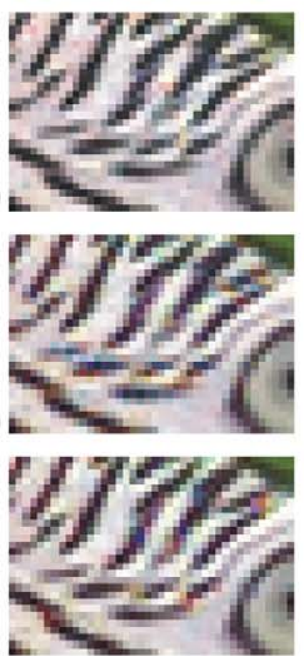
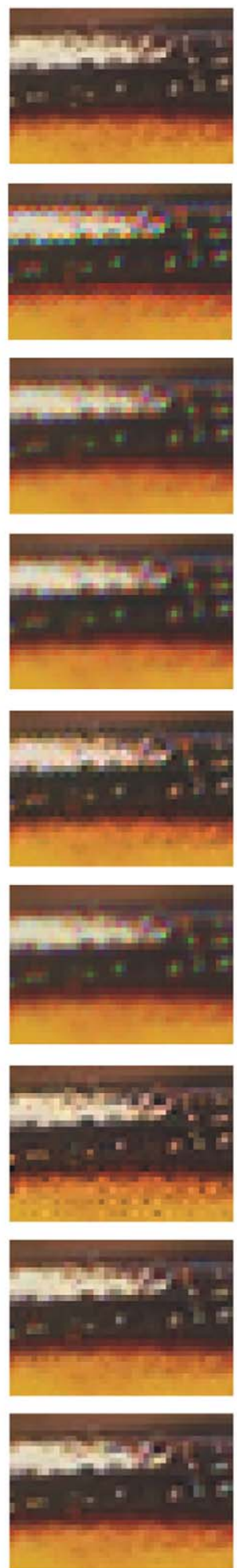
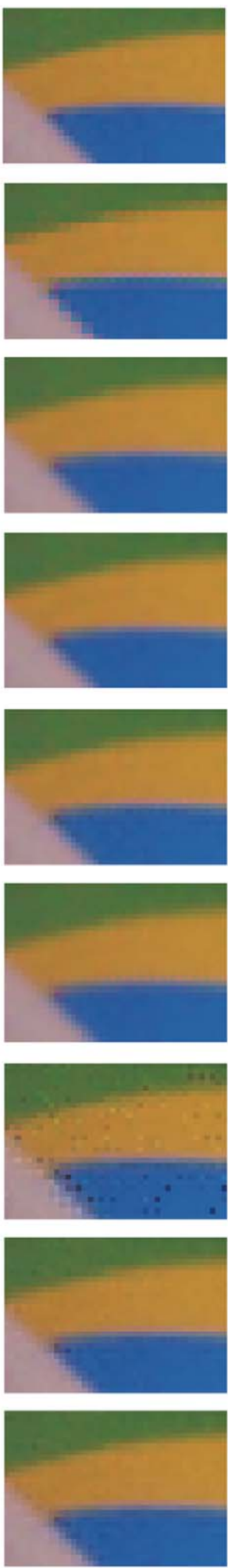
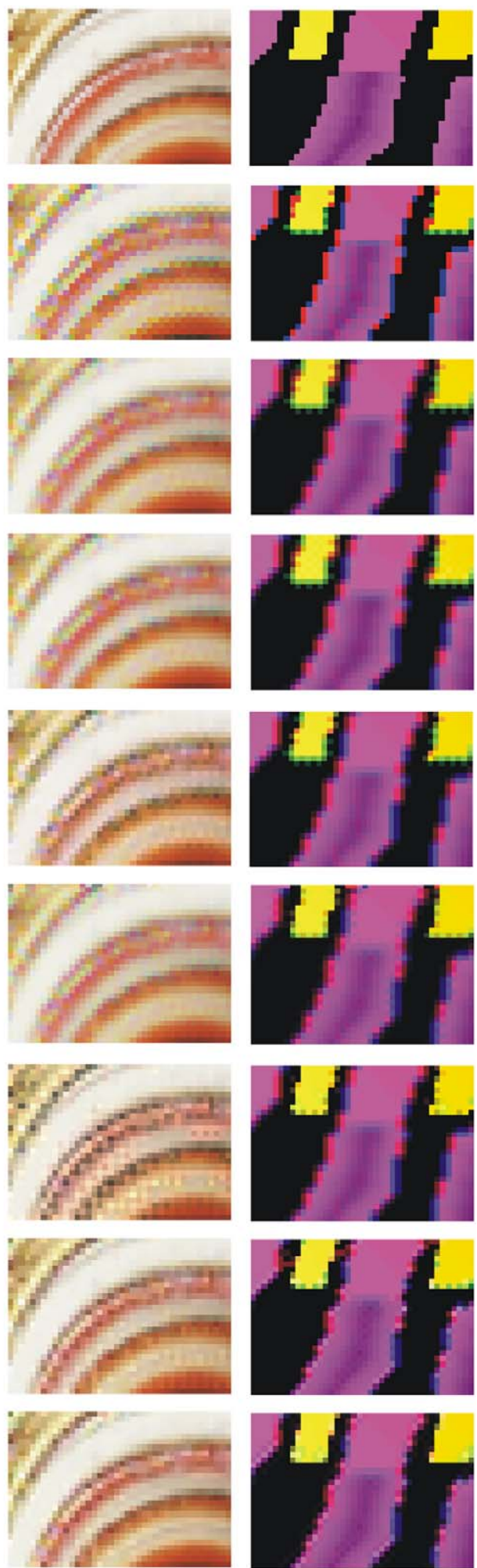

Fig. 14. Zoomed part of the test images and the corresponding results: (a) original images, (b) NNI, (c) BI, (d) BCI, (e) SHT, (f) VMI, (g) SVF, (h) DCCA, (i) MFI, (j) API. 
(a) $\mathrm{i}$

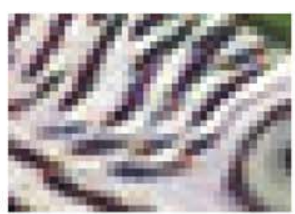

(b)

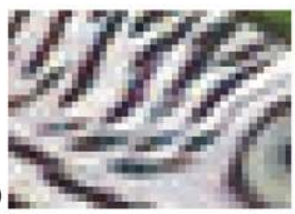

(c) $\mathrm{t}$

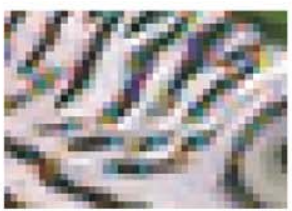

(d)

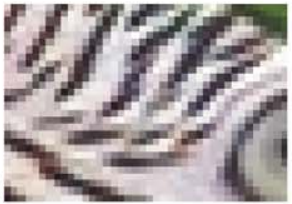

(e)

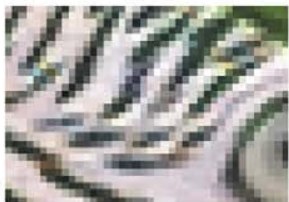

(f)
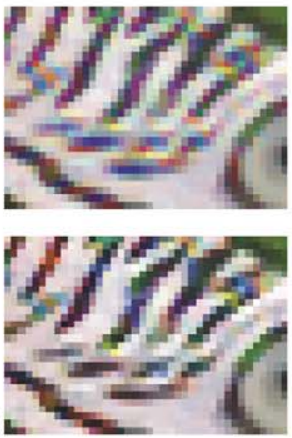

(g)
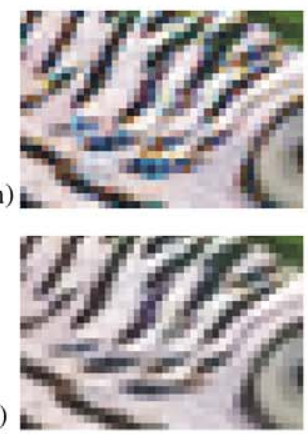
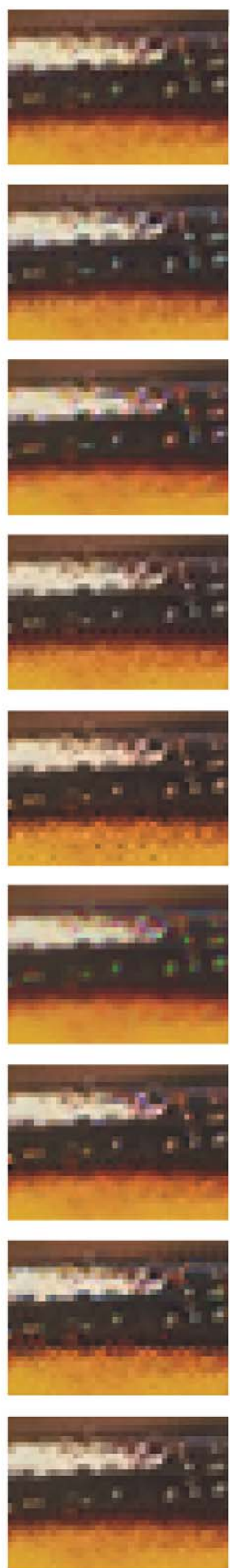
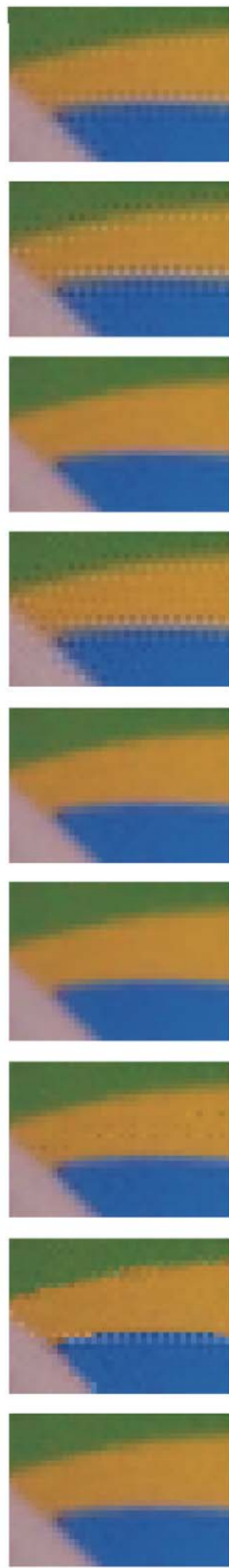
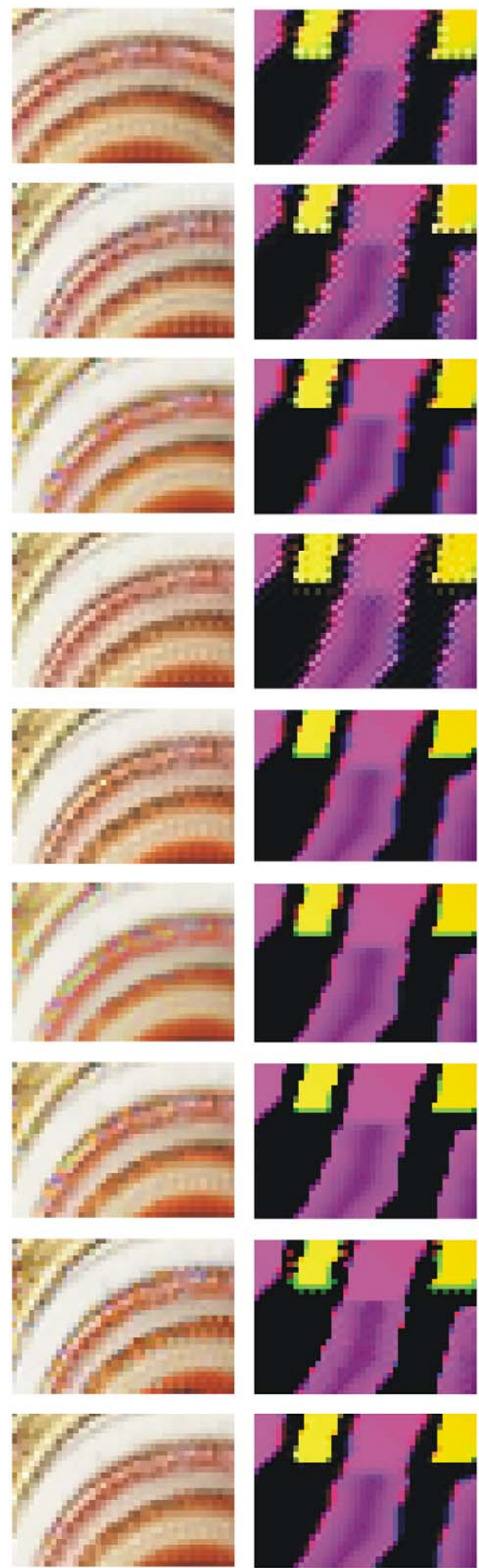

Fig. 15. Zoomed part of the test images and the corresponding results (continued): (a) BD, (b) TDA, (c) PVM, (d) AP, (e) KA, (f) C2D2, (g) SAI, (h) EMI, (i) LIM, (j) proposed ESCC framework. 
(a)

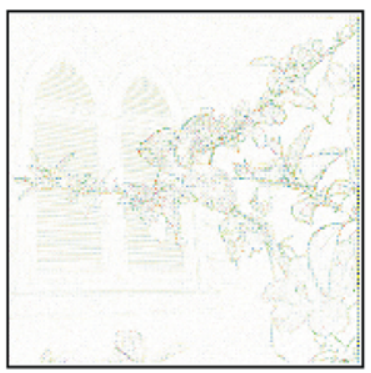

(b)

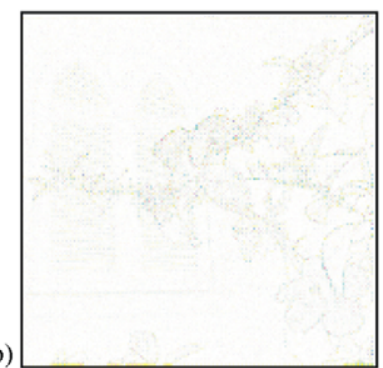

(c)
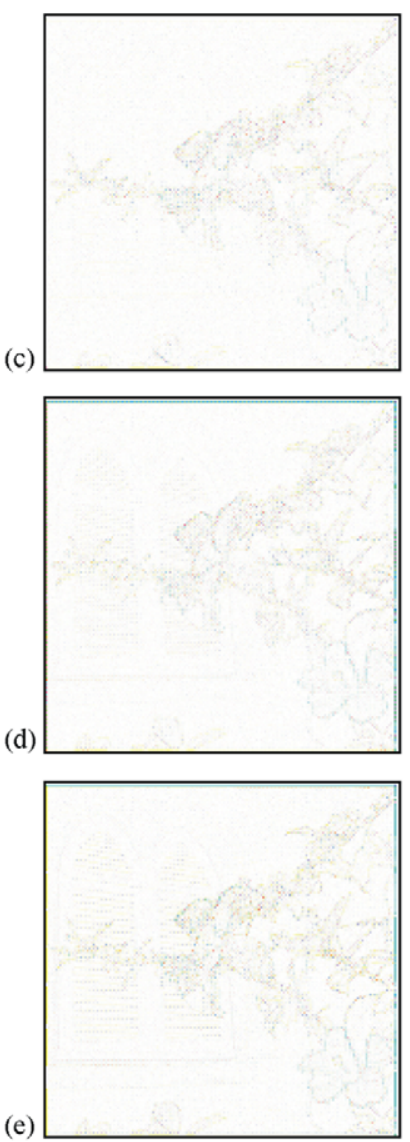
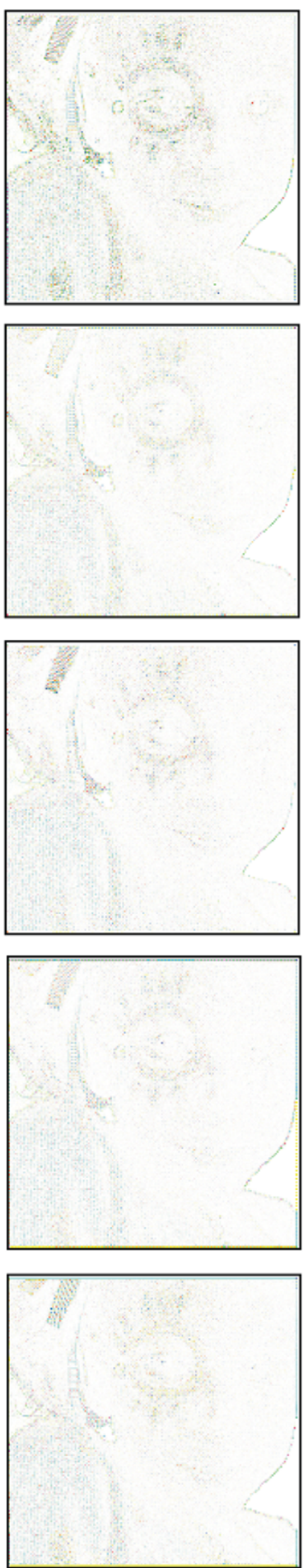
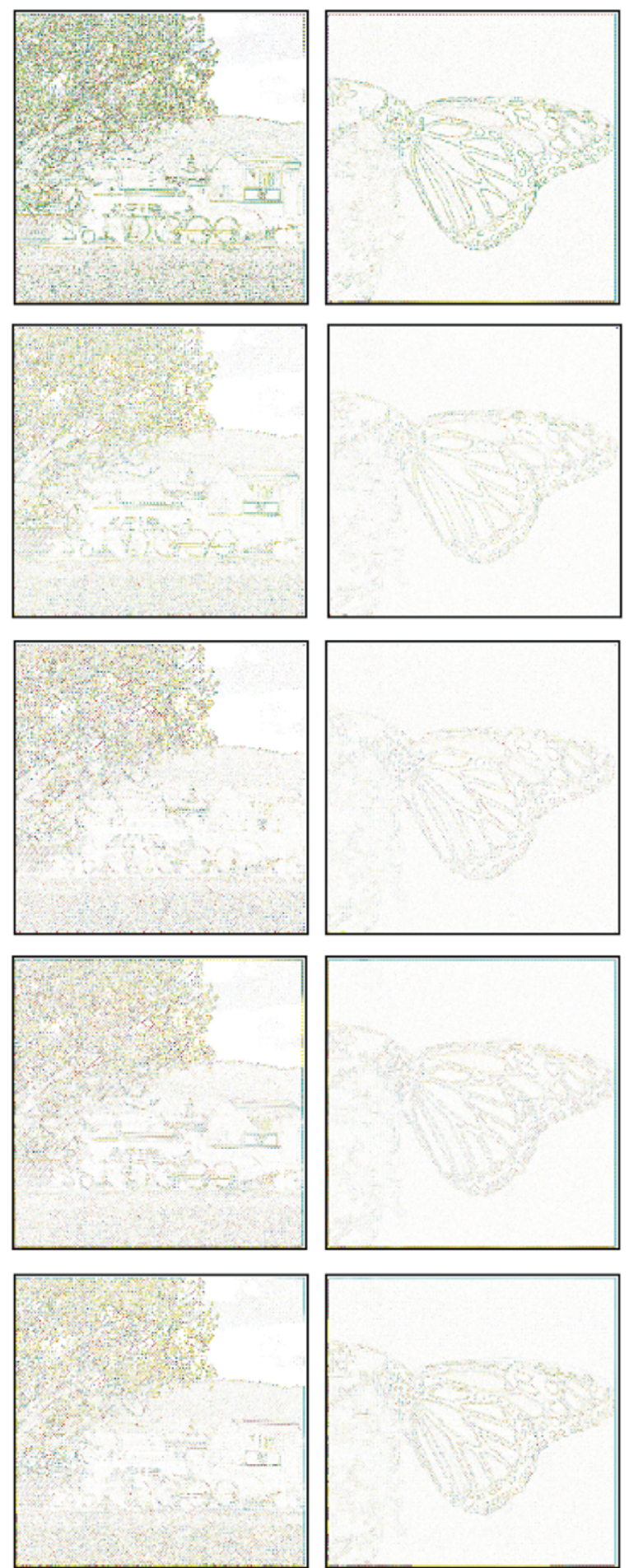

Fig. 16. Estimation errors of interpolation techniques emphasized by a factor of 3: (a) SVF, (b) MFI, (c) API, (d) BD, (e) TDA. 
(a)

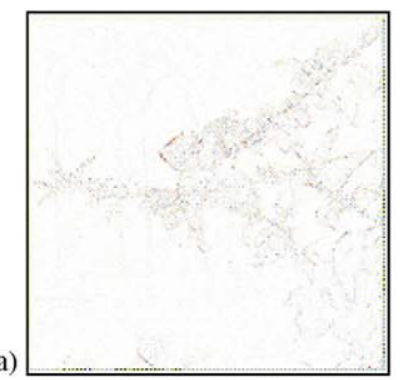

(b)

(c)
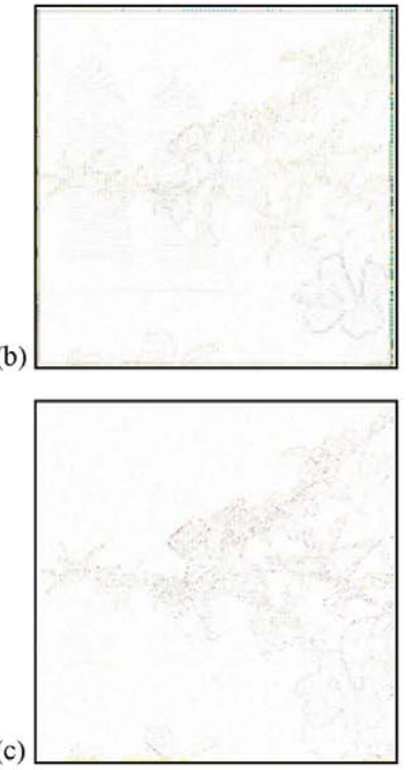

(d)

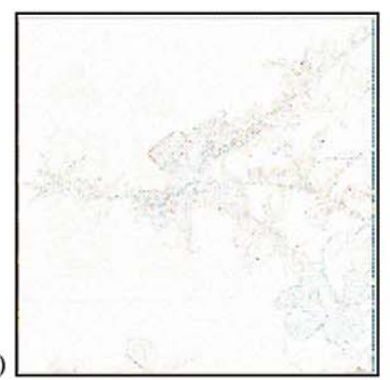

(e)

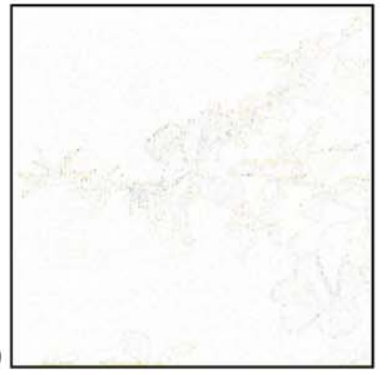

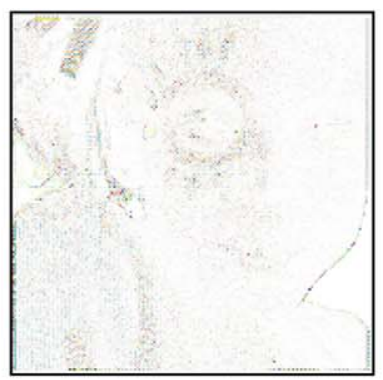
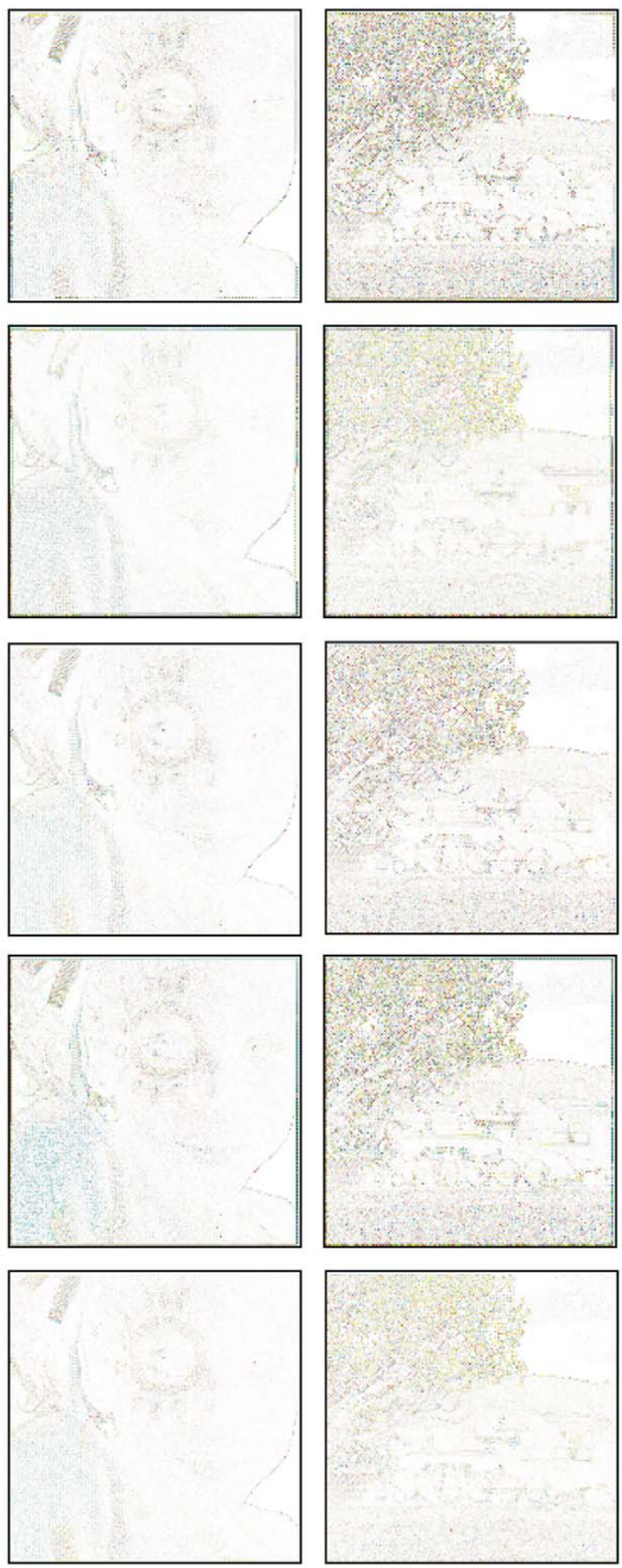
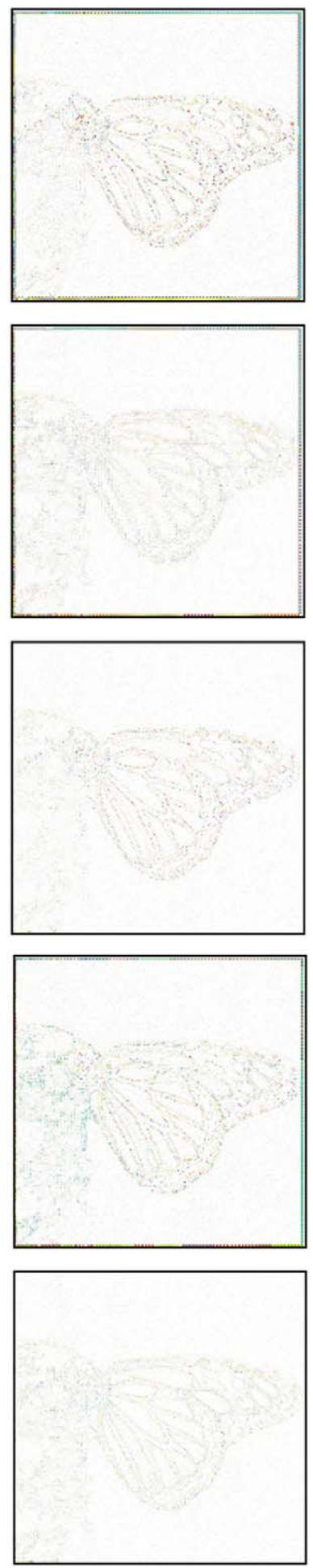

Fig. 17. Estimation errors of interpolation techniques emphasized by a factor of 3: (a) PVM, (b) AP, (c) C2D2, (d) EMI, (e) proposed GESCC framework. 
Table 7

Averaged results related to image database shown in Fig. 12

\begin{tabular}{|c|c|c|c|c|c|c|c|}
\hline Method & $\mathrm{MSE}_{\mathrm{R}}$ & $\mathrm{MSE}_{\mathrm{G}}$ & $\mathrm{MSE}_{\mathrm{B}}$ & $\mathrm{PSNR}_{\mathrm{R}}$ & $\mathrm{PSNR}_{\mathrm{G}}$ & $\mathrm{PSNR}_{\mathrm{B}}$ & $\mathrm{NCD}$ \\
\hline NNI & 398.92 & 152.12 & 475.08 & 24.36 & 28.88 & 24.15 & 0.1252 \\
\hline $\mathrm{BI}$ & 199.70 & 90.43 & 235.41 & 28.20 & 31.73 & 27.83 & 0.0898 \\
\hline BCI & 201.57 & 86.50 & 241.86 & 28.37 & 32.12 & 27.92 & 0.0923 \\
\hline SHT & 302.75 & 90.43 & 241.17 & 27.79 & 31.73 & 28.50 & 0.0866 \\
\hline VMI & 348.68 & 268.20 & 387.07 & 25.16 & 26.49 & 25.05 & 0.1082 \\
\hline SVF & 168.85 & 69.33 & 201.10 & 28.54 & 32.82 & 28.19 & 0.0854 \\
\hline DCCA & 199.53 & 103.91 & 132.31 & 27.94 & 31.78 & 30.77 & 0.0775 \\
\hline MFI & 99.32 & 37.40 & 122.79 & 32.09 & 36.64 & 31.81 & 0.0651 \\
\hline API & 95.48 & 49.57 & 113.23 & 32.72 & 35.43 & 32.64 & 0.0644 \\
\hline $\mathrm{BD}$ & 85.55 & 42.80 & 104.41 & 32.89 & 35.74 & 32.61 & 0.0652 \\
\hline TDA & 103.50 & 53.46 & 126.48 & 31.61 & 34.35 & 30.97 & 0.0713 \\
\hline PVM & 109.06 & 75.26 & 134.55 & 31.62 & 32.85 & 31.27 & 0.0708 \\
\hline $\mathrm{AP}$ & 89.40 & 44.11 & 101.75 & 33.03 & 35.87 & 32.92 & 0.0636 \\
\hline KA & 127.66 & 66.32 & 135.39 & 30.18 & 33.41 & 31.14 & 0.0675 \\
\hline C2D2 & 76.23 & 47.63 & 97.61 & 33.48 & 35.21 & 33.10 & 0.0594 \\
\hline SAIG & 151.96 & 57.31 & 182.79 & 29.66 & 34.16 & 29.25 & 0.0788 \\
\hline EMI & 150.49 & 60.29 & 135.08 & 29.34 & 33.45 & 30.93 & 0.0709 \\
\hline LIM & 151.41 & 62.76 & 183.81 & 29.57 & 33.92 & 29.58 & 0.0747 \\
\hline ESCC & 71.95 & 32.62 & 87.12 & 33.96 & 37.28 & 33.76 & 0.0560 \\
\hline GESCC & 71.78 & 31.24 & 85.67 & 34.08 & 37.50 & 33.94 & 0.0562 \\
\hline LESCC & 71.66 & 31.35 & 85.73 & 34.09 & 37.49 & 33.94 & 0.0562 \\
\hline
\end{tabular}

Table 8

Comparison of the average computational time

\begin{tabular}{|c|c|c|c|c|c|c|c|c|c|}
\hline $\begin{array}{l}\text { Method } \\
\text { time [s] }\end{array}$ & $\begin{array}{l}\text { KA } \\
18.416\end{array}$ & $\begin{array}{l}\mathrm{C} 2 \mathrm{D} 2 \\
5.009\end{array}$ & $\begin{array}{l}\text { PVM } \\
15.383\end{array}$ & $\begin{array}{l}\text { AP } \\
17.229\end{array}$ & $\begin{array}{l}\text { SVF } \\
20.947\end{array}$ & $\begin{array}{l}\text { EMI } \\
26.060\end{array}$ & $\begin{array}{l}\text { ESCC } \\
11.533\end{array}$ & $\begin{array}{l}\text { GESCC } \\
14.384\end{array}$ & $\begin{array}{l}\text { LESCC } \\
17.019\end{array}$ \\
\hline
\end{tabular}

computationally most attractive cases among the considered solutions.

Summarizing the results presented above, the following conclusions can be drawn:

- The proposed method improves color appearance and avoids edge blurring.

- There is no perceptual difference in color appearance between the original image and the images restored by the proposed method.

- The proposed method outperforms significantly the state of-the-art CFA interpolation methods in terms of both objective (more than $20 \%$ ) and subjective image evaluation criteria.

- The proposed method is computationally efficient and compares favorably, in terms of computational complexity, with the widely ;used high-powerful CFA interpolation methods.

\section{Conclusions}

A novel CFA interpolation approach was introduced and analyzed in this work. The method employed color correlations in edge detection and a color correction based CFA interpolation step. Combining their advantages, our method produces interpolated images that are sharp, naturally colored and pleasurable for viewing. At the same time yields excellent results in terms of commonly used objective image quality criteria. Simulation results and comparisons reported here indicate that our framework is sufficiently robust and significantly outperforms previously developed approaches. 


\section{References}

[1] R. Lukac, B. Smolka, K. Martin, K.N. Plataniotis, A.N. Venetsanopulos, Vector filtering for color imaging, IEEE Signal Process. Mag. 22 (1) (January 2005) 74-86 (special issue on Color Image Processing).

[2] K.N. Plataniotis, A.N. Venetsanopoulos, Color Image Processing and Applications, Springer, Berlin, June 2000.

[3] G. Sharma, H.J. Trussell, Digital color imaging, IEEE Trans. Image Process. 6 (7) (July 1997) 901-932.

[4] R. Lukac, K.N. Plataniotis, D. Haztinakos, M. Aleksic, A novel cost effective demosaicing approach, IEEE Trans. Consumer Electron. 50 (1) (February 2004) 256-261.

[5] K.A. Parulski, Color filters and processing alternatives for one-chip cameras, IEEE Trans. Electron Devices ED-32 (8) (August 1985) 1381-1389.

[6] B. Gunturk, Y. Altunbasak, R. Mersereau, Color plane interpolation using alternating projections, IEEE Trans. Image Process. 11 (9) (September 2002) 997-1013.

[7] B.E. Bayer, Color imaging array, US Patent 3971 065, July 1976.

[8] D.R. Cok, Reconstruction of CCD images using template matching, Proceedings of the 47th Annual Conference Imaging Science \& Technology, 1994, pp. 380-385.

[9] W.T. Freeman, Median filter for reconstructing missing color samples, US Patent 4724 395, February 1988.

[10] S.C. Pei, I.K. Tam, Effective color interpolation in CCD color filter arrays using signal correlation, IEEE Trans. Circuits Syst. Video Technol. 13 (6) (June 2003) 503-513.

[11] J.F. Hamilton, J.E. Adams, Adaptive color plane interpolation in single sensor color electronic camera, US Patent $5629734,1997$.

[12] R. Kakarala, Z. Baharav, Adaptive demosaicing with the principal vector method, IEEE Trans. Consumer Electron. 48 (4) (November 2002) 932-937.

[13] R. Kimmel, Demosaicing: image reconstruction from color CCD samples, IEEE Trans. Image Process. 8 (9) (September 1999) 1221-1228.

[14] J. Allebach, P.W. Wong, Edge-directed interpolation, Proceedings of IEEE Conference on Image Processing, vol. 3, September 1996, pp. 707-710.

[15] X. Li, M.T. Orchard, New edge-directed interpolation, IEEE Trans. Image Process. 10 (10) (October 2001) $1521-1527$.

[16] O. Au, M.S. Fu, Spatial varying filtering for color filter array interpolation in digital still cameras, Proceedings of the IEEE International Symposium on Circuits and Systems (ISCAS'03), vol. 2, May 2003, pp. 440-443.

[17] N. Kehtarnavaz, H.J. Oh, Y. Yoo, Color filter array interpolation using color correlations and directional derivatives, J. Electron. Imaging 12 (4) (October 2003) 621-632.

[18] J. Adams, Design of practical color filter array interpolation algorithms for digital cameras, Proceedings of the SPIE, vol. 3028, February 1997, pp. 117-125.

[19] M.R. Gupta, T. Chen, Vector color filter array demosaicing, Proceedings of the SPIE, vol. 4306, May 2001, pp. 374-382.
[20] B. Lee, Performance comparison of various interpolation methods for color filter arrays, Proceedings of the IEEE International Symposium on Industrial Electronics (ISIE'01), vol. 1, June 2001, pp. 232-236.

[21] Y.T. Tsai, W.G. Hsu, S.Y. Tseng, S.L. Cheng, Optimized image processing algorithms for a single sensor camera, Proceedings of the IEEE Pacific Conference on Communications, Computers and Signal Processing, vol. 2, August 1997, pp. 1010-1013.

[22] D.R. Cok, Signal processing method and apparatus for producing interpolated chrominance values in a sampled color image signal, US Patent 4642 678, February 1987.

[23] R. Lukac, K.N. Plataniotis, Normalized color-ratio modeling for CFA interpolation, IEEE Trans. Consumer Electron. 50 (2) (May 2004) 737-745.

[24] P. Longere, X. Zhang, P.B. Delahunt, D.H. Brainard, Perceptual assessment of demosaicing algorithm performance, Proc. IEEE 90 (1) (January 2002) 123-132.

[25] R. Ramanath, W.E. Snyder, G.L. Bilbro, W.A. Sander, Demosaicking methods for the Bayer color array, J. Electron. Imaging 11 (3) (July 2002) 306-315.

[26] T. Sakamoto, C. Nakanishi, T. Hase, Software pixel interpolation for digital still cameras suitable for a 32-bit MCU, IEEE Trans. Consumer Electron. 44 (4) (November 1998) 1342-1352.

[27] C. Cai, T.H. Yu, S.K. Mitra, Saturation-based adaptive inverse gradient interpolation for Bayer pattern images, IEE Proc.-Vision, Image, Signal Process. 148 (3) (June 2001) 202-208.

[28] B.S. Hur, M.G. Kang, High definition color interpolation scheme for progressive scan CCD image sensor, IEEE Trans. Consumer Electron. 47 (1) (February 2001) 179-186.

[29] H.J. Trussell, R.E. Hartwig, Mathematics for demosaicking, IEEE Trans. Image Process. 11 (4) (April 2002) 485-492.

[30] W. Lu, Y.P. Tan, Layering-based color filter array interpolation, Proceedings of the IEEE International Conference on Image Processing (ICIP'01), vol. 3, October 2001, pp. $860-863$.

[31] T. Kuno, H. Sugiura, New interpolation method using discriminated color correlation for digital still cameras, IEEE Trans. Consumer Electron. 45 (1) (February 1999) 259-267.

[32] R. Lukac, K. Martin, K.N. Plataniotis, Demosaicked image postprocessing using local color ratios, IEEE Trans. Circuits Syst. Video Technol. 14 (6) (June 2004) 914-920.

[33] H. Zen, T. Koizumi, H. Yamamoto, I. Kimura, H. Hashizume, A new digital signal processor for progressive scan CCD consumer, Proceedings of the International Conference on Consumer Electronics (ICCE'97), June 1997, pp. $350-351$.

[34] R.G. Keys, Cubic convolution interpolation for digital image processing, IEEE Trans. Acoustics, Speech Signal Process. 29 (6) (December 1981) 1153-1160.

[35] D. Su, P. Wilis, Demosaicing of colour images using pixel level data-dependent triangulation, Proceedings of the International Conference on Theory and Practice in Computer Graphics, June 2003, pp. 16-23. 